\title{
When do we know that we do not know? An Examination of Metacognitive Processes in Visual Working Memory
}

\author{
Julia Krasnoff \& Klaus Oberauer \\ University of Zurich, Cognitive Psychology
}

Note: This manuscript is a pre-print.

It may not replicate the final publication of this work.

\begin{abstract}
Author Note
The project was funded by the Swiss National Science Foundation (grant number 100014 179002). The data and analysis scripts of all experiments are available over the Open Science Framework (see: https://osf.io/5mgzt/). The study material is available on request from the corresponding author. The study was not preregistered.

We thank Marlies Monch, Florian Eschenmoser, and Victoria Häffner for helping with data collection.
\end{abstract}

Correspondence should be addressed to Julia Krasnoff, Department of Psychology, Cognitive Psychology Unit, University of Zurich, Binzmuehlestrasse 14/22, 8050 Zurich, Switzerland. E-mail: j.krasnoff@psychologie.uzh.ch. 


\begin{abstract}
This work investigates how people make judgements about the content of their visual working memory (VWM). Some studies on long-term memory suggest that people base those metacognitive judgements on the outcome of a retrieval attempt. In contrast, Son and Metcalfe (2005) observed that people identify poorly remembered items immediately, presumably by the lack of familiarity for the retrieval cue. We tested these two hypotheses in the context of metacognition in VWM. In Experiment 1-3, we investigated participants' response behavior in a color reproduction task with a hidden color wheel, which partially externalizes the search for the intended response. We assumed that instant awareness of the inability to retrieve an information would be reflected in selecting the first, random color, rather than search for a particular color in the wheel. Although participants provided a substantial number of low-confidence responses, results of an adapted mixture modelling analysis yielded little evidence for quick guesses. In contrast, participants consistently searched for a color (even with unfamiliar retrieval cues in Experiment 2), and only quickly guessed when being cued with objects at test that were not previously presented (Experiment 3). In Experiment 4, we tested whether the order of color reproduction and confidence judgements influences reaction times (RTs) for these tasks. We observed shorter RTs for whichever response was given second, as would be expected if reproduction and confidence judgments shared a retrieval attempt. We conclude that people usually engage in retrieval attempts for providing judgements about their VWM, even when information is poorly remembered.
\end{abstract}

Keywords: visual working memory, guessing, confidence ratings, mixture model 


\section{When do we know that we do not know? An Examination of Metacognitive Processes in Visual Working Memory}

Everyone who has completed a test most likely experienced the feeling of not knowing the correct response to a question. In this situation, being instantly aware of one's inability to retrieve the correct response - instead of engaging in a long unsuccessful retrieval attempt first would save some valuable time that could be strategically allocated to more promising test questions. A substantial body of research on long-term memory (LTM) has investigated whether people possess such an instant insight into their memory and how, in general, people make judgements about their memory content (also referred to as metacognitive judgements). Although there are many studies on metacognition in LTM, little is known about how people make judgements about the content of their working memory (WM). WM differs from LTM in several aspects: It is capacity-limited, holds information only briefly active for further processing, and is sensitive to experimental manipulations that do not affect LTM (e.g., Brady et al., 2013). Therefore, it is also reasonable to assume differences in how people make judgements about the content of their WM and LTM, respectively. The aim of the present work is to provide new insights into metacognitive processes in WM. More specifically, we aim to find out how people judge which information they can, and which information they cannot retrieve from their WM.

Because there is only little research on metacognition in WM, we have drawn from the literature on metacognition in LTM as a starting point for the present study. Research on metacognition in LTM mainly offers two hypotheses on how people know that they do not know some information. The first hypothesis is that people base their metacognitive judgements on the outcome of a retrieval attempt (Begg et al., 1989; Benjamin et al., 1998; Kelley \& Lindsay, 1993; Nelson \& Dunlosky, 1991). In the following, we refer to this idea as the retrieval-outcome 
hypothesis. Proponents of the retrieval-outcome hypothesis argue that people can only know that they do not remember something after failing to retrieve it from memory. This idea is supported by modelling results that show longer reaction times (RTs) for "guessed" responses as compared to "retrieved" responses (Province \& Rouder, 2012) and likewise by experimental findings showing that wrong responses in recognition tests are associated with longer RTs than accurate responses (Donkin et al., 2013). Longer RTs for wrong responses speak in favor of the retrievaloutcome hypothesis because they imply that participants do not immediately select a random answer when seeing the question but instead engaged in a process that takes at least as long as a successful retrieval process before choosing a (usually wrong) response.

In contrast with the retrieval-outcome idea and the aforementioned findings, Son and Metcalfe (2005) propose that people do not always need to engage in a retrieval attempt for making a judgement about their memory content. In their experiments, the authors asked participants in the first step to memorize first name-last name pairs. In the second step, participants were cued with the first name and asked to give a judgement of learning (JOL), a prediction about the probability of retrieving the information in a future test, for the corresponding first name-last name pair. The authors then varied whether participants were explicitly instructed to retrieve the last name prior to giving the JOL, or were solely instructed to give a JOL, and investigated the relation between JOLs and RTs in these two conditions. They found that the relation between JOL and RT largely depended on the condition. In the condition where participants were explicitly instructed to engage in a retrieval attempt prior to giving a JOL, findings were consistent with previous findings on the correlation between RTs and the correctness of the responses: RTs for retrieval + JOL were longer when JOLs were lower (when participants predicted that an item will likely not be remembered in the future) than for items 
with higher JOLs (when participants predicted that an item will be likely remembered).

However, when participants were solely instructed to make a JOL, a large proportion of very low JOL responses were given relatively fast, implying that no retrieval attempt has taken place. The latter finding therefore challenged the retrieval-outcome hypothesis that predicts longer RTs for lower JOLs independent of experimental instructions. Son and Metcalfe (2005) concluded that there must be a two-stage process for generating JOLs: In a first stage people judge the familiarity of the cue that they are given (in their experiments, the first name), and if this cue fails to reach a certain familiarity threshold, no retrieval takes place and people assign a very low JOL to the corresponding item. If, in contrast, the cue exceeds the familiarity threshold, people engage in a retrieval attempt and base their JOL on its outcome, as is assumed in the retrievaloutcome hypothesis.

Here we provide the first test of the retrieval-attempt-hypothesis and the two-stage process hypothesis in the context of metacognitive judgements in WM. We aim to determine whether people need to engage in a retrieval attempt for making a judgement about their WM content. For that purpose, we make use of an experimental procedure and analysis method that stems from research on visual working memory (VWM). Previous studies investigating the topic of metacognitive judgements mainly used verbal material and recognition or cued recall tasks (for a meta-analysis see Double et al., 2018). Consequently, conclusions about the underlying metacognitive processes were mainly based on RT data and the correctness of the responses. In this study, we used a color reproduction task that allows us to not only look at RT data but also observe participants' behavior while searching for a response. Our procedure provides a new method for investigating whether metacognitive judgments are based on a rapid assessment of 
familiarity, as postulated by the two-stage process (Son \& Metcalfe, 2005), or can be made solely on the basis of a retrieval attempt.

Besides providing novel insights into metacognitive processes in WM, this study can provide some additional insights into the nature of WM and its limited capacity. To date there is no consensus on what limits WM. Some theorists argue that the limited capacity of WM can be described as a discrete number of storage units (sometimes called slots), each of which can hold one item or chunk (e.g., Cowan, 2001; Zhang \& Luck, 2008). This assumption implies that, when the number of items in a memory set exceeds capacity, some of the items will be maintained in a slot, whereas others will not be represented in memory at all. If this is the case, then it is plausible - though not necessary - to assume that people know which items they have encoded into a slot. When tested on an item not in a slot, they would know right away that they have no chance of retrieving it, and therefore could respond with a fast guess without attempting retrieval.

By contrast, a second class of theories rests on the assumption that a limited resource is divided among all items in a memory set, so that every item is encoded into WM with some strength - although that strength can vary substantially between items even within the same set (e.g., Fougnie et al., 2012; Van Den Berg et al., 2012). If that is the case, the decision for making a fast guess is less straightforward: Even if people have immediate metacognitive insight into the strength of each item in WM, they could never know for sure whether it is futile to try to retrieve a weak item.

According to a third kind of theory, access to items in WM is primarily limited by interference (e.g., Oberauer \& Lin, 2017). In these models, all items of a memory set are encoded and maintained strongly, and memory errors arise from mutual distortions of representations in 
WM, and from confusions of the target item with other items, and with noise, at retrieval. If these theories are correct, there is no obvious way for a person to decide whether a particular item will be successfully retrieved without trying. To conclude, finding out whether people sometimes know that they do not know an item in WM cannot decisively rule out one or another class of theories, but it can render them more or less plausible.

\section{Experiment 1}

The aim of Experiment 1 was to investigate whether people possess an instant knowledge about their inability to retrieve a visual feature (here, a color) from VWM by analyzing participants' response behavior during the test phase of a color reproduction task. The general idea was to create a response scale on which participants had to overtly search for an intended color, whereas a random guess could be given without such a search. Participants had to select a color from a color wheel that was covered, and only the color underneath the current mouse position was revealed at any time point. We assumed that participants who know upfront that they will not be able to retrieve the target color will not spend time searching for the correct color - neither mentally, nor physically in the color wheel. On the contrary, the most efficient strategy in that case is to select as one's response the very first random color that is revealed once the mouse hits the color wheel, and proceed to the next, possibly more promising trial. However, such an efficient strategy would only be possible if participants had an instant insight in their inability to retrieve the target information. If a retrieval attempt is necessary to conclude that no retrieval is possible, participants should engage in a mental, and possibly also physical search for the correct response prior to guessing. 


\section{Method}

Participants. 20 students from the University of Zurich participated in this lab experiment for either 15 CHF or partial course credit. Only students between the age of 18-35 years without any impairment of their color vision were recruited for this experiment.

Materials and Procedure. The experiment was programmed in MATLAB using the Psychophysics Toolbox extension (Brainard, 1997). Participants completed 3 practice trials, followed by 324 experimental trials. The experimental procedure is depicted in Figure 1.

\section{Figure 1}

Procedure in Experiment 1.

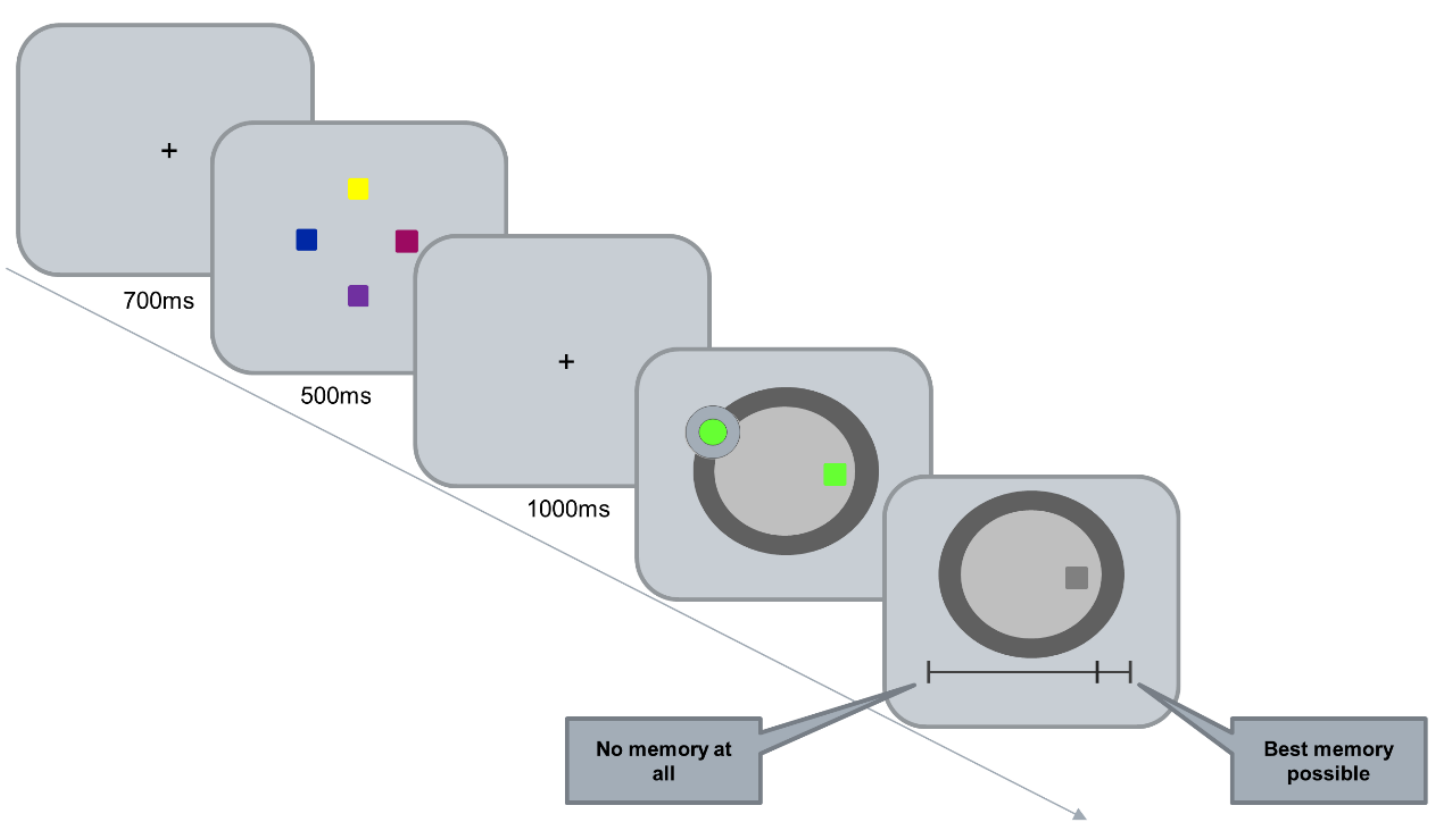

Note. Flow of events in a single experimental trial of Experiment 1. This example shows a set size four trial.

Each trial started with a fixation cross that appeared for $700 \mathrm{~ms}$ in the middle of the screen. Participants were then presented with either two, four or eight colored squares (set size manipulation) simultaneously for $500 \mathrm{~ms}$. Colors were randomly sampled from a color circle in 
the CIELAB color space $(L=70, a=20, b=38$, and radius $=60)$. After the colored squares were presented, another fixation cross appeared in the screen center for one second. Following this retention interval, participants were randomly cued with the location of one of the presented squares and had to reproduce its color on a "greyed" color wheel, that is, a dark grey circle centered on the screen center covering an invisible color wheel (see Souza et al., 2016). The main property of a grey wheel - as compared to a color wheel - is that participants do not directly see all colors of the wheel. Only by moving the cursor into and along the wheel, the underlying colors become visible. The underlying color wheel rotated randomly on each trial, making it impossible to know the position of the colors underneath the grey wheel. Instead, participants always must enter the grey wheel with the mouse first and then search the position of the correct color from there by moving the mouse along the grey band. Upon finding the desired color, participants selected it as their response by a mouse click. After that, they were asked to judge their confidence in the given response. A continuous linear scale appeared ranging from "no memory at all" to "best memory possible", and participants were asked to answer the question "How well is your memory for this color?" by shifting the slider with the mouse to the corresponding position. The slider only appeared after participants moved the cursor along the scale.

The study material is available on request from the corresponding author. The data and analysis scripts of all experiments are available over the Open Science Framework (see: https://osf.io/5mgzt/). 


\section{Results}

Descriptives. Figure 2 shows the distributions of confidence, and of the deviation of the selected color from the correct response, over all participants for the different set size conditions. Whereas confidence judgments in the upper half of the scale were more frequent, there were also observations that fell into the very low confidence range. This was especially the case in the set size eight condition. A comparable pattern can be observed with the deviation: Whereas most deviations were close to 0 , speaking for participants' strong performance, some very large errors were also observed, especially in the set size eight condition. Thus, even though highly confident, and accurate responses dominated, participants showed substantial variance in their confidence and performance. As expected, confidence and recall error (the absolute deviation of the given color from the correct color) were on average correlated ${ }^{1}$ to $r=.42$, suggesting that participants have some insights into their VWM content.

${ }^{1}$ To quantify the average correlation between recall error and confidence judgements, Spearman's correlation coefficients were computed for each participant individually, they were then transformed to Fisher's z scale and averaged across all participants, before being transformed back to Spearman's r scale. 


\section{Figure 2}

Frequency of Confidence Judgements and Deviation in Experiment 1 as a Function of Set Size.

frequency of deviation
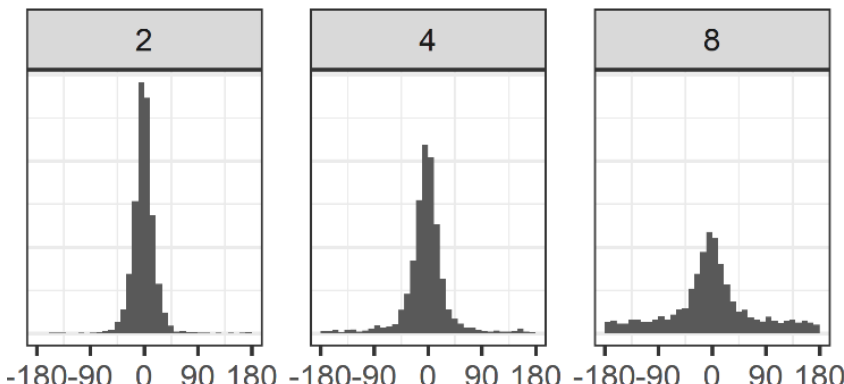

deviation

frequency of confidence responses
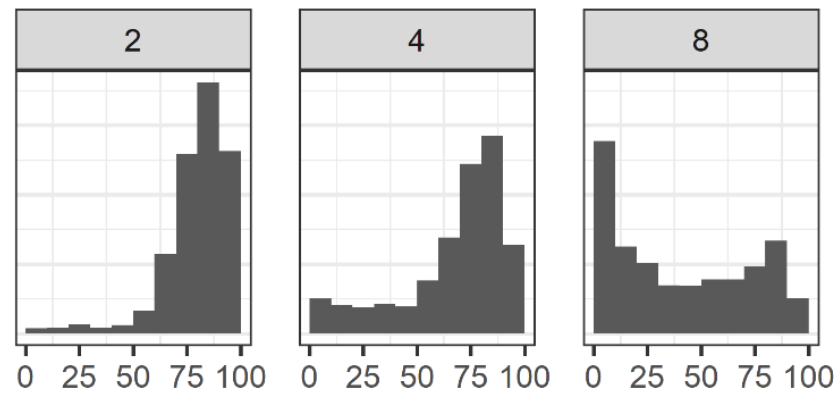

confidence

Our main dependent variable in this experiment was the angle between the first random color that appeared when touching the grey wheel with the mouse cursor, and the given response. In the following, we will refer to this angle as the search angle. An average search angle of 90 degrees is expected when the given response is at a random distance from the first color. Because the correct response and the first color hit by the mouse are independent random draws from the color wheel, the expected search angle for correct responses is 90 . More generally, responses distributed symmetrically around the correct response would yield an average search angle of 90 . Therefore, a mean search angle of 90 is predicted if people's responses were guided by memory independent of their starting point in the wheel. In contrast, an average angle of 0 would occur when the first random color was always selected as the response. Therefore, fast guessing with 
little search for a particular color would lead to search angles close to zero. Figure 3 depicts the average serach angle as a function of confidence.

\section{Figure 3}

Search Angle as a Function of Confidence.

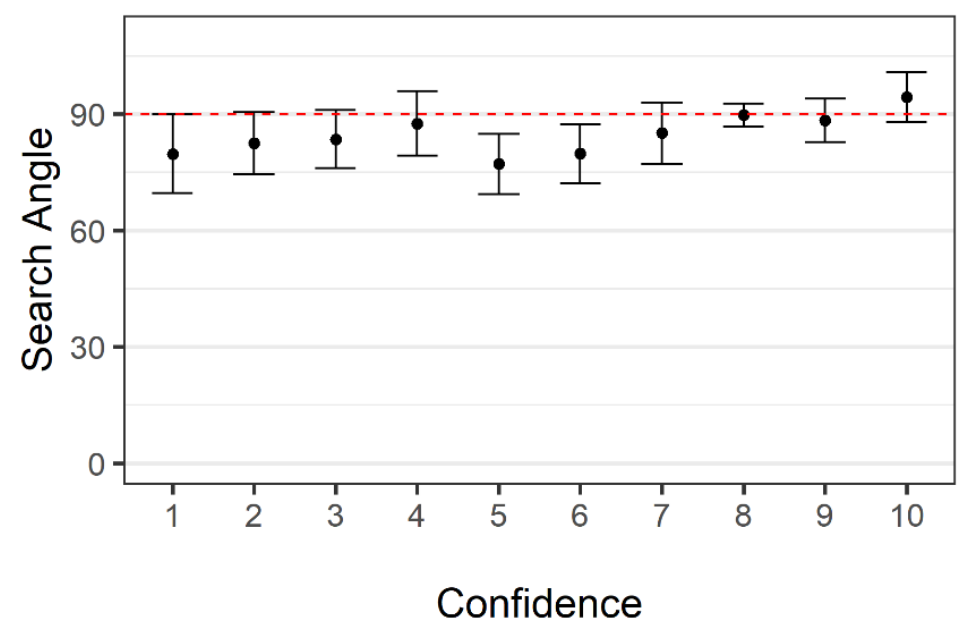

Note. Error bars represent 95\% within-subjects confidence intervals. The dashed line marks the average search angle under the assumption that responses are at a random distance from the first color. The continuous confidence scale was binned in 10 bins ranging from 1 (very low confidence) to 10 (very high confidence).

In some confidence bins the search angle was smaller than 90, providing evidence for some quick, random responses. However, the average search angle was still overall very high and thus, far away from a value of 0 at all levels of confidence. This shows that participants' responses were, for the most part, at a random distance from the first color, and that selecting the first random color was rather the exception, even in the lowest confidence bins. 
Modelling. We tested the assumption that people's search for a response in the grey wheel is governed by one of two metacognitive states: Believing that one knows the correct color (at least approximately), and believing that one has no idea, and therefore must give a guess. We assume that awareness of having zero information about the color in memory would be reflected in selecting a response close to the first random color appearing when touching the grey wheel. This is because there is no reason to waste time on a search process that one already knows to be futile. This, in turn, will manifest in a search angle of (close to) zero between the first random color and the selected response. When aggregating over several trials, the distribution of search angles in this metacognitive state of conscious guessing results in a von Mises distribution that peaks around a search angle of zero. In the following we refer to this distribution as the guessingawareness distribution. In contrast, we assume that people search for a color in the wheel when they believe to have some information available about the color. While on some trials, the correct response will be by chance close to the first random color, on other trials it will be far away from it. Because the orientation of the color wheel is drawn from a uniform distribution on the circle on each trial, and the correct response is independently drawn from a uniform distribution, the distance between them is also uniformly distributed. Therefore, aggregating responses from this metacognitive state will result in a uniform distribution of the search angle over all possible values: the retrieval-attempt distribution.

To investigate whether there is evidence for these two metacognitive states, we adapted the mixture model from Zhang and Luck (2008) and applied the adapted model to participants' response behavior data. Figure 3 shows the original mixture model and our adapted version of it. Both models are a mixture of a uniform and a von Mises distribution and thus have two free parameters. One parameter is $K$, describing the width of the von Mises distribution. The second 
parameter is $P$ and it describes the probability of the observed angle coming from the von Mises distribution. However, there are two crucial differences between theses versions of the mixture model. The first difference is that the original model describes the distribution of recall errors (see left plot in Figure 4), whereas the adapted model describes the distribution of the search angles (see right plot in Figure 4). The second difference is that the original model assumes two distinct cognitive states (a memory and a guessing state) that result in two different distributions of the recall error, whereas the adapted version assumes two metacognitive states that result in different response behavior distributions. Therefore, the interpretation of the two parameters differs between the models. Contrary to the original model, where $K$ represents the precision of the memory representations, there is no such substantive interpretation of $K$ in the adapted model. It simply captures participants' response noise when guessing. The main parameter of interest in the adapted model is $P_{g}$ - namely the probability of a search angle coming from the von Mises or guessing-awareness distribution. Thus, one can infer from $P_{g}$ the percentage of trials in which participants are instantly aware of their inability to retrieve the response and react with a quick guess.

If people have immediate metacognitive insight into the availability of a memory representation for the target, then we expect that $P_{g}$ will be rather small at set sizes 2 and 4 (because on most trials participants have some knowledge of the color and therefore will engage in a retrieval attempt) but increase to a substantial value at set size 8 , which far exceeds common estimates of WM capacity (around 3 items): For instance, according to slot models, the majority of set size 8 trials test an item that is not in memory. If people know which items they do, and which ones they do not have represented in a slot, they should give fast guesses on more than half the set size 8 trials. 


\section{Figure 4}

Illustration of the standard Mixture Model for Recall Errors and the adapted Mixture Model for Search Angles.
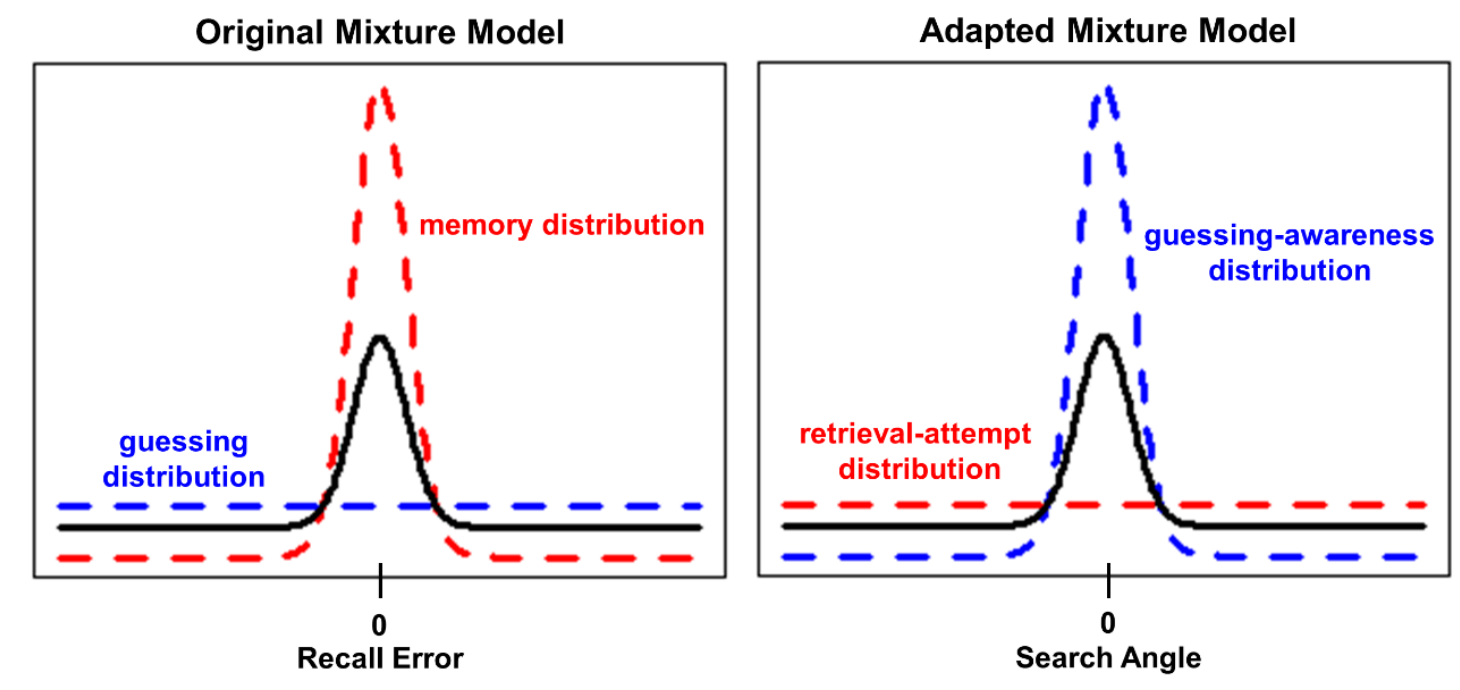

Note. Original mixture model (on the left) describing the observed distribution of recall error as a mixture of a memory and a guessing distribution. Adapted mixture model (on the right) describing the observed distribution of the search angle (first random color - selected color) by a mixture of a guessing-awareness and a retrieval-attempt distribution.

We fitted the adapted mixture model to the search angle data in two different ways. First, as a Bayesian hierarchical model and second, individually to the data of each participant with a maximum-likelihood algorithm. This procedure enabled us to evaluate the models' fit on the level of the whole population and for each participant separately. In both modeling approaches, the lower bound for the parameter $P_{g}$ was set to its natural lower bound of 0 . Likewise, the upper bound for the parameter $P_{g}$ was set to its natural ceiling of 1 . We decided to set the lower bound of the parameter $K$ to 3 to ensure that the von Mises distribution stays distinguishable from a 
uniform distribution ${ }^{2}$. Additionally, to avoid numerical problems we set the upper bound for $K$ to 100, which corresponds to a very small variance. To examine whether there is evidence for quick guesses, we next compared the adapted mixture model to a pure uniform model. In other words, we tested whether including the von Mises distribution representing guessing-awareness improves the model fit, or whether the search angle data can similarly well be described by just the uniform distribution representing retrieval attempts. In the Bayesian hierarchical approach, we computed the Watanabe Akaike Information Criterion (WAIC) for both models for each set size condition. We then compared the models' fits by computing the difference between the

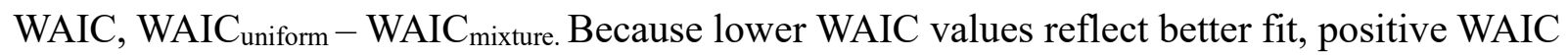
differences reflect more support for the mixture model, and negative WAIC differences reflect more support for the uniform model. We proceeded in the same way when fitting the models to the data of each participant individually. For the latter approach, the Akaike Information Criterion (AIC) was computed. Table 1 shows the results of the Bayesian hierarchical modelling approach.

\section{Table 1}

Mean Posterior Parameter Estimates of the Bayesian Hierarchical Adapted Mixture Model, WAIC for the Adapted Mixture Model (WAIC mix $)$, the Uniform Model (WAIC uni) and Difference in WAIC (WAIC uni- WAIC $_{\text {mix }}$ ) for Data of Experiment 1 as a Function of Set Size

\begin{tabular}{cccccc}
\hline set size & $P_{g}$ & $K$ & WAIC $_{\text {mix }}$ & WAIC $_{\text {uni }}$ & $\begin{array}{c}\text { WAIC }_{\text {uni- }} \\
\text { WAIC }_{\text {mix }}\end{array}$ \\
\hline 2 & .021 & 14.36 & 7936.94 & 7939.63 & 2.69 \\
& {$[0, .046]$} & {$[3.0,37.76]$} & & & \\
4 & .018 & 9.88 & 7939.41 & 7939.63 & 0.22 \\
& {$[0, .043]$} & {$[3.0,24.21]$} & & & \\
8 & .111 & 7.44 & 7846.45 & 7939.63 & 93.18 \\
& {$[.064, .16]$} & {$[3.0,14.62]$} & & & \\
\hline
\end{tabular}

${ }^{2}$ If $K=0$, the van Mises distribution corresponds to a uniform distribution. 
Note. Values in brackets represent the boundaries of the $95 \%$ highest density interval for the parameters $P_{g}$ and $K$.

The differences in WAIC were positive in all set size conditions, reflecting a benefit of the mixture model over the uniform model. Therefore, on the level of the whole population there was evidence for quick guesses in all set size conditions. However, the probability to be in the guessing-awareness distribution, $P_{g}$, was estimated to be between $2 \%$ and $11 \%$ in all conditions. Assuming a capacity limit of around 3 items, such a low probability of quick guesses is to be expected in the set size two condition, but it falls far below the expected probability in the set size eight condition where, according to a slot model, participants must have guessed in around half of the trials. Therefore, if people were in a guessing state as often as predicted by a slot model for the set size eight condition, they were rarely aware of their inability to retrieve the color in such a state before trying to retrieve it. Additionally, the $K$ parameter was estimated to be rather low in all conditions, reflecting a high width of the guessing-awareness distribution. Therefore, even when being in the guessing-awareness distribution participants did not select the first color, but rather moved around a bit in the wheel before abbreviating their search and clicking on a color. Taken together, the hierarchical modelling approach provided some evidence for quick guesses, but their occurrence was much lower than what would be expected on the basis of a slot model if people always knew right away that they will have to guess.

Whereas fitting the model hierarchically offers the benefit of examining the fit of the models on the level of the whole population, it does not provide information on how well the models fit to the data of individual participants. Therefore, we additionally fit the model separately to the data of each participant. Figure 5 shows the estimates of $P_{g}$ across set sizes averaged over participants. The probability to be in the guessing-awareness distribution was 
rather small for all participants in all conditions, replicating the results from the hierarchical modelling approach. As expected, mean $P_{g}$ was higher in set size eight as compared to set sizes two and four, which can be explained by the increased difficulty of the task. Nevertheless, mean $P_{g}$ remained far lower than the number of responses that participants must have guessed at set size eight if their capacity was limited to about 3 to 4 slots. This result is consistent with the result of the hierarchical modelling approach.

\section{Figure 5}

Estimates of the Probability to be in the Guessing-Awareness Distribution $\left(P_{g}\right)$ as a Function of Set Size in Experiment 1.

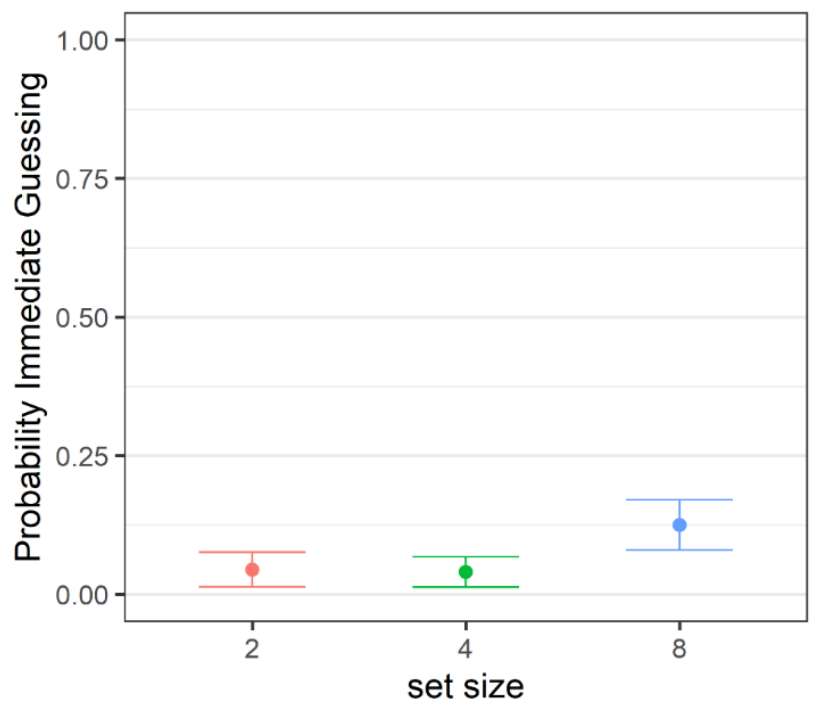

Note. Error bars represent $95 \%$ within-subjects confidence intervals.

Next, we computed the AIC for the mixture and the uniform models for each participant. The differences in AIC are depicted in Figure 6: For most participants and conditions, the difference in the goodness of fit for the two models was close to 0 . This shows that the uniform model provided a similarly good fit to the data as the adapted mixture model for most of the 
participants $^{3}$. Only for a small subset of participants in some conditions the mixture model showed an advantage: Most participants in most conditions seemed to search for the color in the grey wheel before giving their guess.

Figure 6

Frequencies of AIC Differences in Experiment 1.

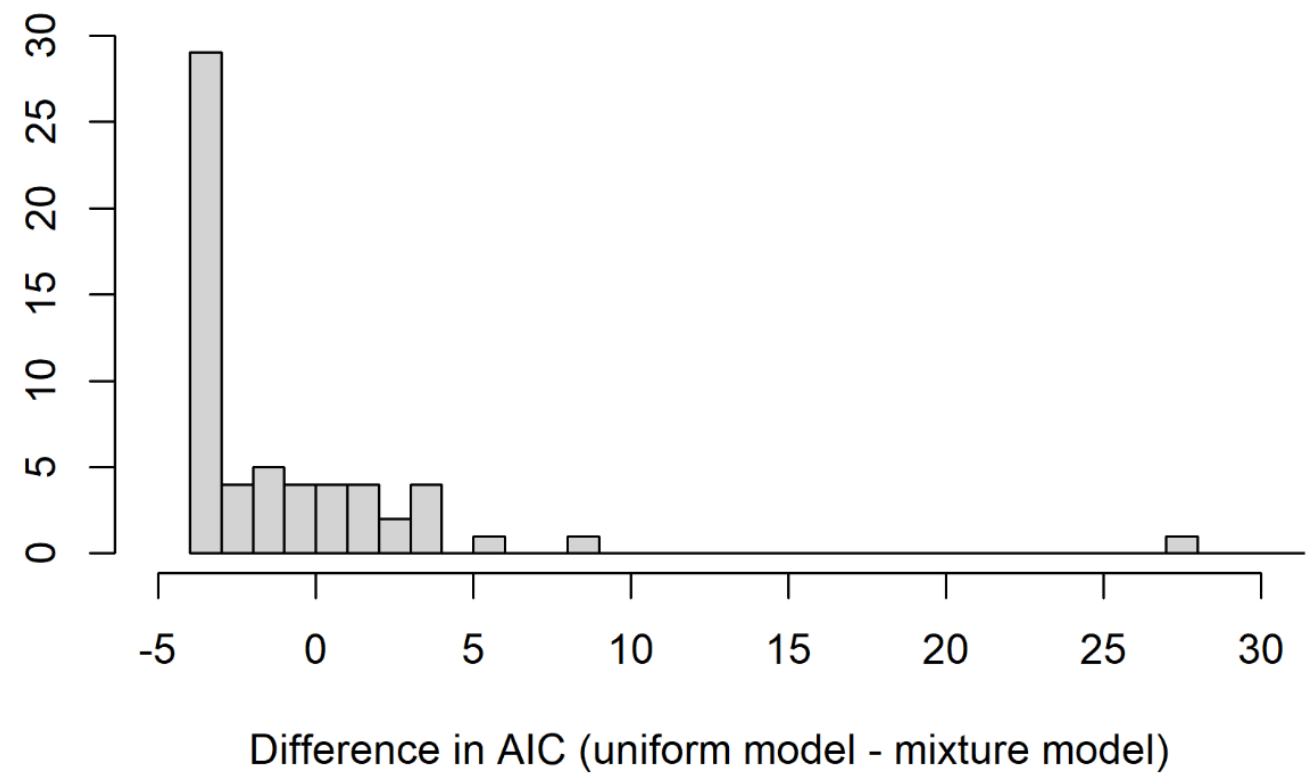

Note. Positive values show an advantage of the mixture model over the uniform model, and negative values show an advantage of the uniform model.

\section{Discussion}

In Experiment 1, we tested whether people possess an instant knowledge about their inability to retrieve a color from WM, or whether they must engage in a retrieval attempt to realize that they do not remember the color. The use of a grey wheel in Experiment 1 enabled us

${ }^{3}$ The $K$ parameter was estimated to be close to its defined limits ( 3 and 100) for some of the participants in some conditions. This was probably due to the few observations in the data that provided information about the von Mises distribution. 
to monitor how long people searched for the desired response, offering an additional measure for participants' metacognitive states besides the commonly used RTs. Results of this experiment provided little evidence for the tendency to select the first random response. Such a behavior only occurred in a very small number of trials and only for some of the participants - suggesting that most participants were not aware right away of their inability to retrieve an accurate response. Moreover, the finding that the average search angle was close to 90 even in lowconfidence trials, speaks in favor of the retrieval-outcome-hypothesis for metacognitive processes in VWM. Most of the time people seem to engage in a retrieval attempt before realizing that they can give only a response with high uncertainty, or an outright guess.

One limitation of the present experiment lies in the type of retrieval cue that was used. Son and Metcalfe (2005) argued that a judgment of not knowing without a retrieval attempt only occurs when the retrieval cue is unfamiliar. In Experiment 1, however, participants were cued with one of eight possible locations that were used repeatedly throughout the experiment. Thus, the retrieval cues could all have chronically reached the familiarity threshold, preventing participants to ever encounter a sufficiently unfamiliar cue to come to a quick judgment of not knowing. Experiment 2 was conducted to test this possibility.

\section{Experiment 2}

The aim of Experiment 2 was to test whether there is evidence for quick guesses when participants are confronted with less familiar retrieval cues. To examine this, participants were presented with two, four or eight colored objects that appeared simultaneously in different positions of the screen. In contrast to Experiment 1, where participants were cued by position, participants were cued by the object in Experiment 2. Importantly, half of the objects were presented repeatedly whereas the other half of the objects was only presented once in a single 
trial. Thus, Experiment 2 allowed us to investigate participants' response behavior as a function of the familiarity of the retrieval cue, and test whether there is evidence for quick guesses when the retrieval cue is less familiar.

\section{Method}

Participants. 81 students from the University of Zurich participated in this online experiment for either 7.50 CHF or partial course credit. Only students between the age of 18-35 years and without any impairment of their color vision were recruited.

Materials and Procedure. The experiment was programmed in lab.js (Henninger et al., 2019). Participants completed 3 practice trials, followed by 150 experimental trials. The experimental procedure is depicted in Figure 7.

\section{Figure 7}

Procedure in Experiment 2.

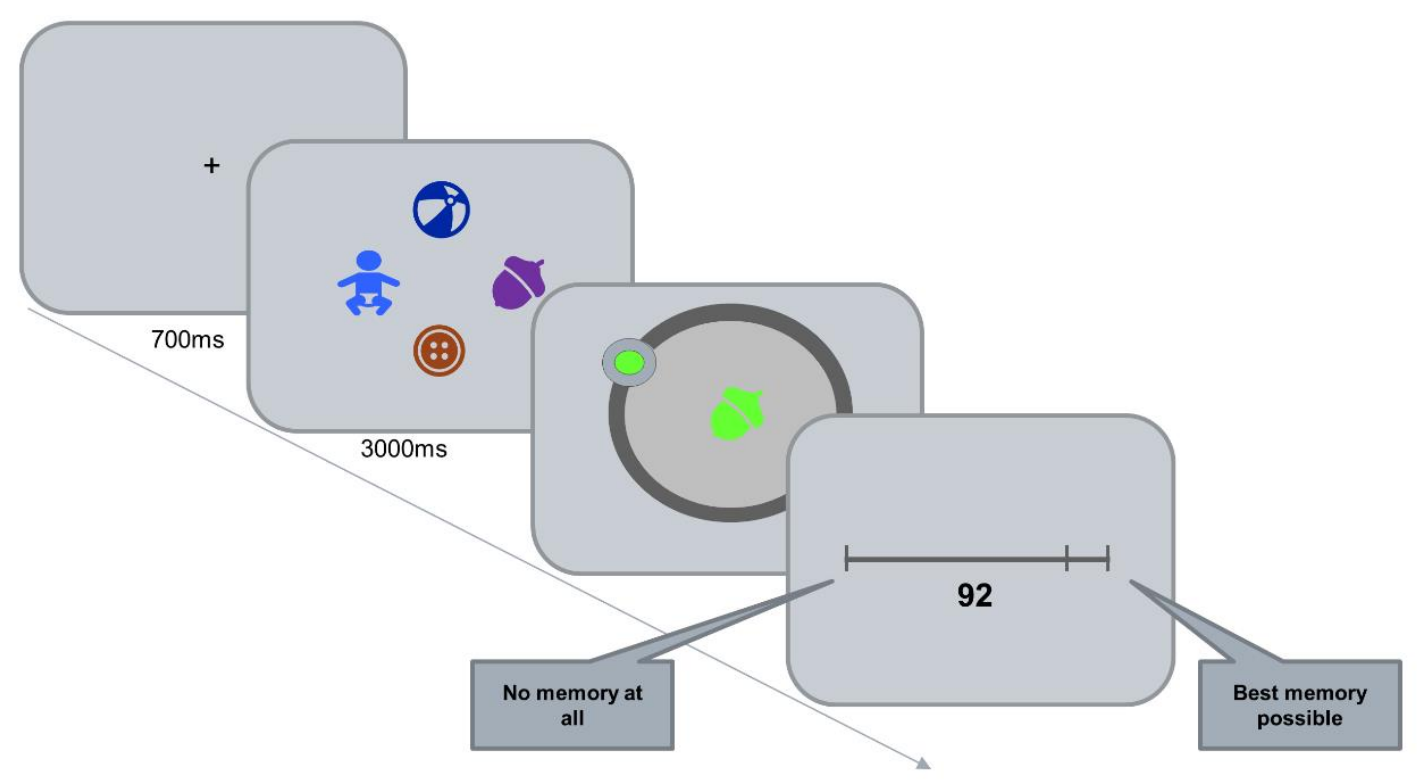

Note. Flow of events in a single experimental trial of Experiment 2. This example shows a set size four trial. The number below the slider represented the confidence rating that corresponds to the position of the slider. 
Before the start of the experiment, four objects were randomly drawn from the pool of 431 objects for each participant. These four objects were used as recurring objects throughout the entire experiment. The experimental procedure resembled the procedure in Experiment 1 with the following changes: Participants were presented with either two, four or eight colored objects (set size manipulation) for the duration of $3000 \mathrm{~ms}$ (the presentation duration was extended to ensure encoding of all objects). Half of the objects in each trial were drawn from the four recurring objects, the other half was drawn from the remaining pool of objects. Following encoding, one object was presented in grey color in the middle of the screen. This object was either one of the recurring objects (recurring cue condition) or one of the non-recurring objects (non-recurring cue condition). The rest of the experimental procedure was the same as in Experiment 1 . To check whether the manipulation of familiarity worked, participants were asked at the end of the experiment to indicate whether they realized that half of the objects were presented recurringly (Manipulation Check). 


\section{Results}

Manipulation Check. Twenty-two participants indicated to not have noticed that part of the objects reoccurred on each trial. Therefore, all analyses were conducted with and without these 22 participants. As excluding these participants did not lead to any differences in the pattern of results, the results of the whole sample are reported below.

Descriptives. Figure 8 shows the distributions of confidence and of the deviation of the selected color from the correct color over all participants and both cue conditions. As in Experiment 1, a substantial number of poor responses and of low confidence responses was observed, particularly at the higher set sizes. Again, there was a correlation between confidence judgements and recall error, reflected in an average Spearman's correlation coefficient of $r=$ $-.47$ 


\section{Figure 8}

Frequency of Confidence Judgements and Deviation in Experiment 2 as a Function of Set Size.

frequency of deviation
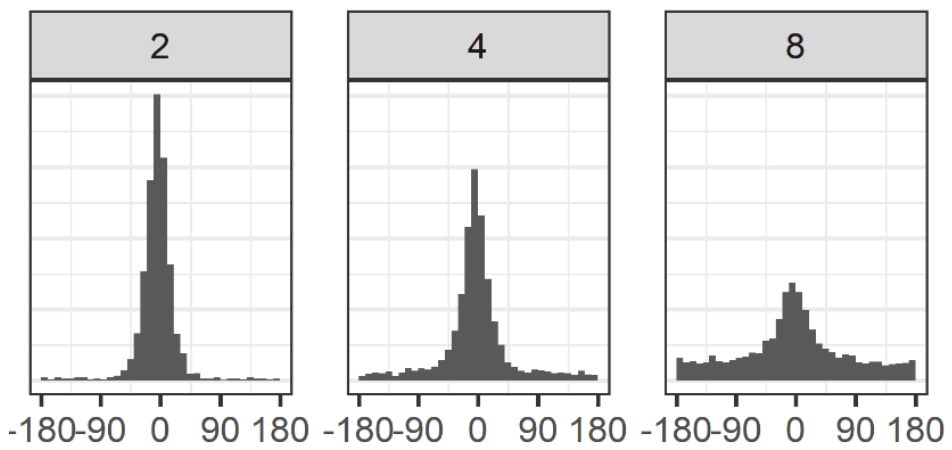

deviation

frequency of confidence responses
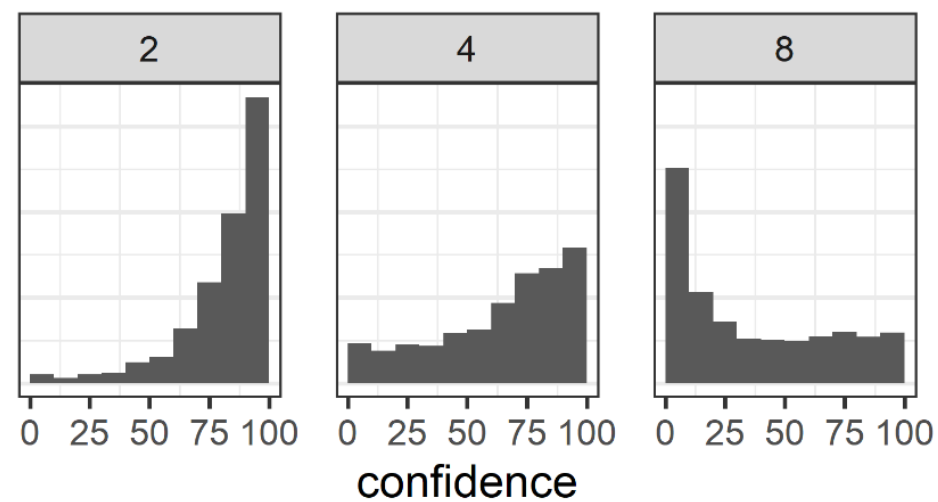

Note. Plot shows the observations of all participants and both cue conditions.

Figure 9 shows the average search angle in both cue conditions as a function of confidence. Again, all means are close to 90, and no systematic differences are apparent between the cue conditions. 


\section{Figure 9}

Search Angle as a Function of Confidence and Cue.

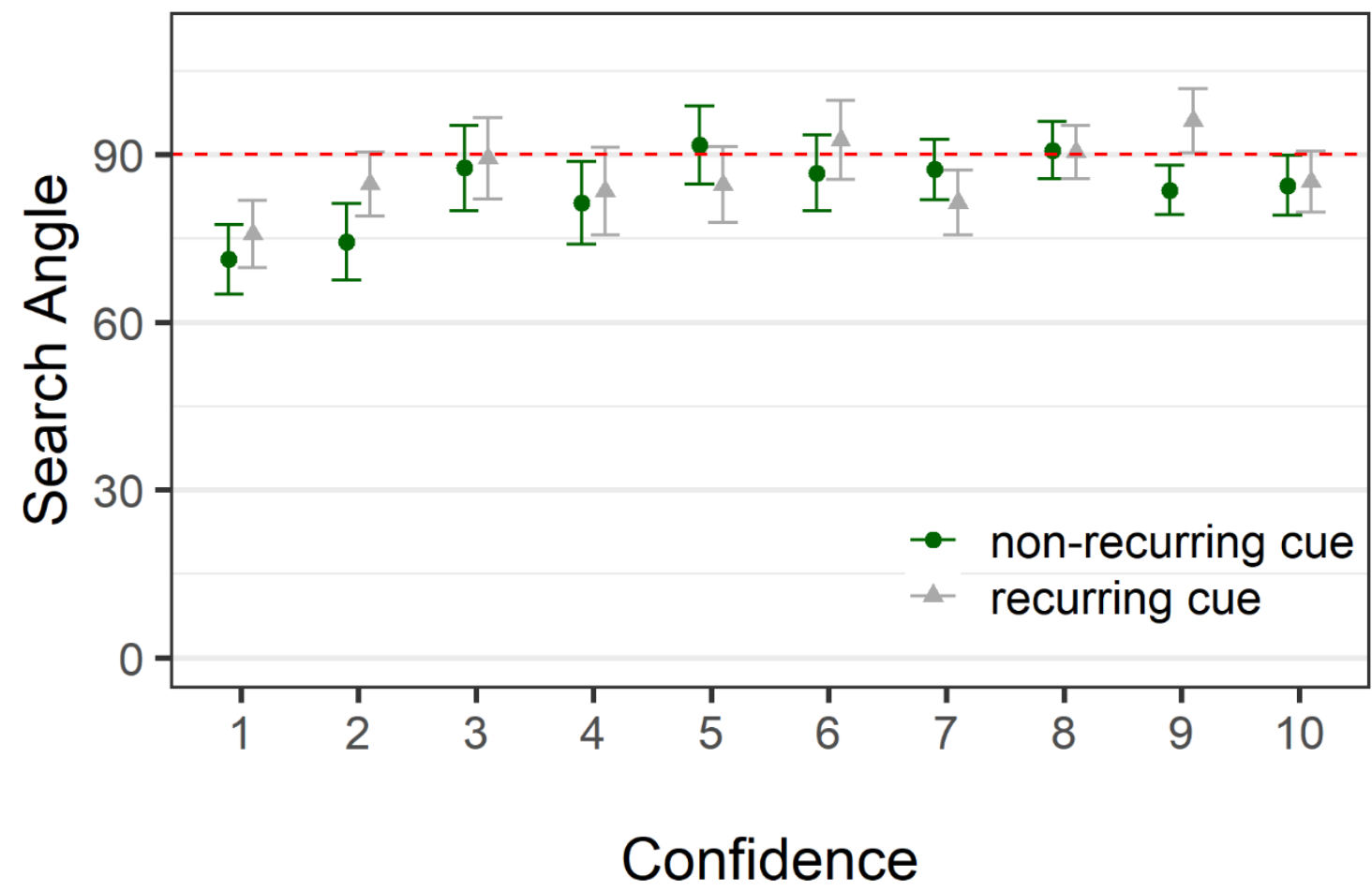

Note. Error bars represent $95 \%$ within-subjects confidence intervals. The dashed line marks the average search angle under the assumption that responses are at random distance from the first color. The continuous confidence scale was binned in 10 bins ranging from 1 (very low in confidence) to 10 (very high in confidence).

Consistent with the findings of Experiment 1, the search angle data deviated from 90 in some of the confidence levels, reflecting a tendency to select a color close to the first random color in some rare cases. However, even in the lowest confidence bin the average search angle was very far away from 0 - indicating that participants moved around the wheel before selecting their response on most of the trials. 
Modelling. To analyze participants' response behavior, we proceeded in the same way as in Experiment 1. We first fitted a Bayesian hierarchical mixture model and the uniform model to the search angles of all participants and estimated the goodness of fit and the parameters of the adapted mixture model for each condition on the level of the whole population (see Table 2). In the second step we fitted the adapted mixture and the uniform model to the data of each participant separately to examine the models' fit on the participants' level. The same limits as in Experiment 1 were set for the two free parameters of the adapted mixture model.

\section{Table 2}

Mean Posterior Parameter Estimates of the Bayesian Hierarchical Adapted Mixture Model, WAIC for the Adapted Mixture Model (WAIC $C_{m i x}$ ), the Uniform Model (WAIC uni) and Difference in WAIC (WAIC uni- WAIC $_{\text {mix }}$ ) for Data of Experiment 2 as a Function of Set Size and Cue Condition

\begin{tabular}{|c|c|c|c|c|c|c|}
\hline cue & $\begin{array}{l}\text { set } \\
\text { size }\end{array}$ & $P_{g}$ & $K$ & $\mathrm{WAIC}_{\text {mix }}$ & WAIC $_{\text {uni }}$ & $\begin{array}{l}\text { WAIC }_{\text {uni- }} \\
\text { WAIC }_{\text {mix }}\end{array}$ \\
\hline \multirow[t]{2}{*}{ recurring } & 2 & $\begin{array}{c}.016 \\
{[0, .037]}\end{array}$ & $\begin{array}{c}9.47 \\
{[3.0,23.04]}\end{array}$ & 7437.08 & 7443.4 & 6.32 \\
\hline & 4 & $\begin{array}{c}.027 \\
{[0, .062]}\end{array}$ & $\begin{array}{c}11.18 \\
{[3.0,25.59]}\end{array}$ & 7373.60 & 7443.4 & 69.8 \\
\hline \multirow{4}{*}{$\begin{array}{l}\text { non- } \\
\text { recurring }\end{array}$} & 8 & $\begin{array}{c}.089 \\
{[.028, .143]}\end{array}$ & $\begin{array}{c}7.8 \\
{[3.0,15.52]}\end{array}$ & 7300.05 & 7443.4 & 143.35 \\
\hline & 2 & $\begin{array}{c}.027 \\
{[0, .062]}\end{array}$ & $\begin{array}{c}7.31 \\
{[3.0,15.5]}\end{array}$ & 7394.86 & 7443.4 & 48.54 \\
\hline & 4 & $\begin{array}{c}.033 \\
{[0, .07]}\end{array}$ & $\begin{array}{c}6.5 \\
{[3.0,12.89]}\end{array}$ & 7402.38 & 7443.4 & 41.02 \\
\hline & 8 & $\begin{array}{c}.071 \\
{[0, .128]}\end{array}$ & $\begin{array}{c}6.05 \\
{[3.0,10.72]}\end{array}$ & 7282.54 & 7443.4 & 160.86 \\
\hline
\end{tabular}

Note. Values in brackets represent the boundaries of the $95 \%$ highest density interval for the parameters $P_{g}$ and $K$.

Table 2 shows the results of the hierarchical modelling approach. The positive difference between the WAIC for the uniform model and the mixture model reflects an advantage of the mixture model over the uniform model in all conditions, replicating the results of Experiment 1. 
As expected, the estimates for $P_{g}$ increased with increasing set size. Although, $P_{g}$ was estimated to be around $7-9 \%$ in the set size eight condition (which is consistent with the results of Experiment 1), it was still lower than what would be expected from a slot model with 3-4 slots, if participants engaged in quick guesses on all trials where they did not have the target item in a slot. In addition, the $K$ parameter was again estimated to be low. This means that even in the guessing-awareness state participants move around a little bit before shortening their search and selecting a response - instead of clicking on the first random color. In contrast with the idea that people can come to a quick judgment of not knowing when encountering a sufficiently unfamiliar retrieval cue, we did not observe any substantial differences in the results between the two cue conditions. Taken together, the results of the hierarchical modeling approach replicated the findings of Experiment 1 and suggested that the unfamiliarity of the retrieval cue does not lead to more quick guesses.

As in Experiment 1, we also fitted both models to the data of each participant separately. The resulting average $P_{g}$ values were again rather low in all conditions, and equally high in the recurring condition as compared to the non-recurring cue condition (see Figure 10). $P_{g}$ was highest in the set size eight condition, again replicating the results of Experiment 1. 


\section{Figure 10}

Estimates of the Probability to be in the Guessing-Awareness Distribution $\left(P_{g}\right)$ as a Function of Set Size and Cue Condition in Experiment 2.

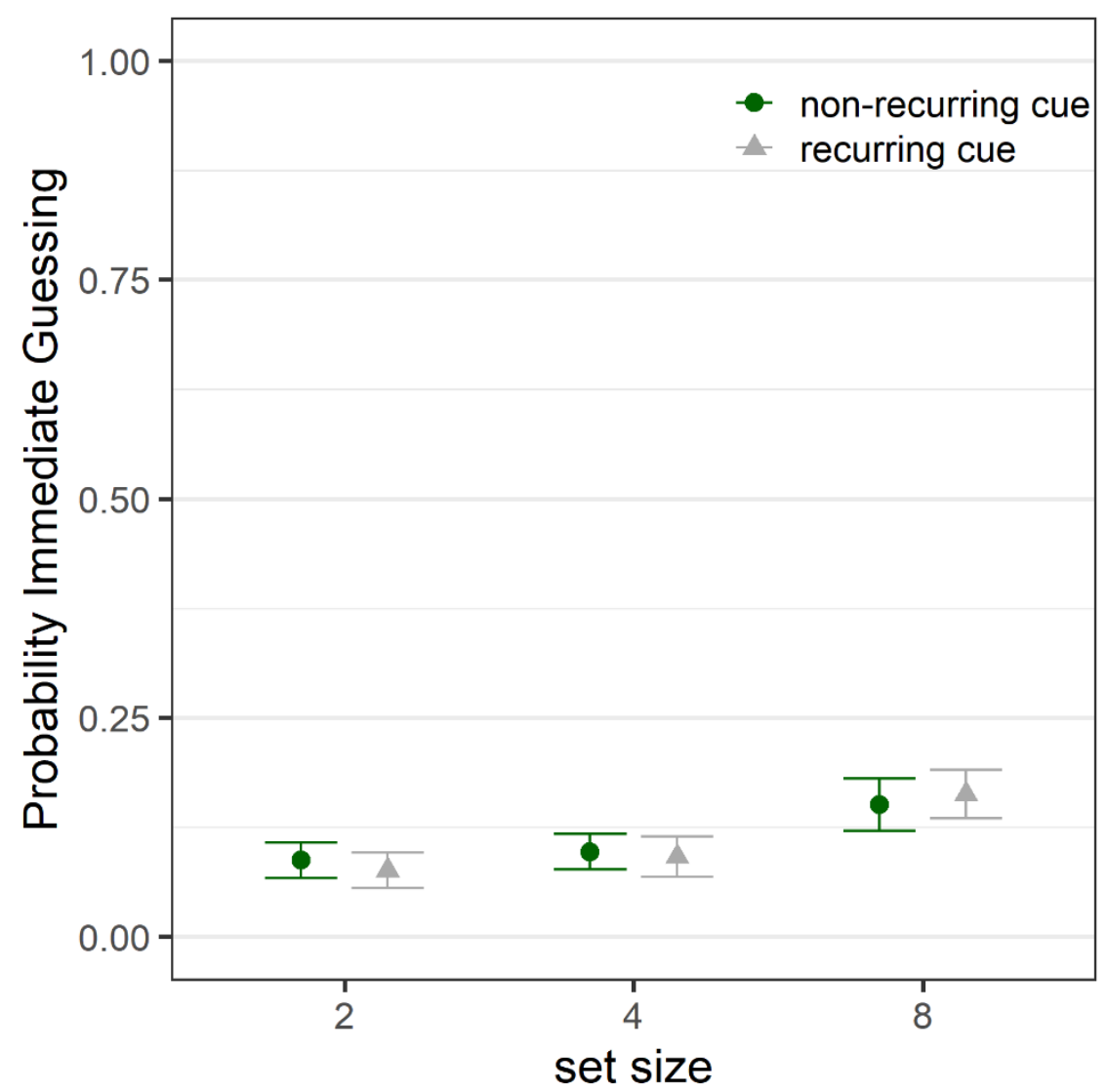

Note. Error bars represent 95\% within-subjects confidence intervals.

A comparison of the AIC between the mixture model and the uniform model for each participant for both the recurring cue and the non-recurring cue condition yielded only sparse evidence for the existence of a guessing-awareness distribution (see Figure 11). In both cue conditions, the AIC for the uniform model indicated a better fit as the AIC for the mixture model for the majority of participants. Hence, the search angle data in both cue conditions can be described by a uniform distribution for most of the participants. 


\section{Figure 11}

Frequencies of AIC Differences in the Recurring Cue Condition (left) and Non-Recurring Cue Condition (right).

Recurring Cue

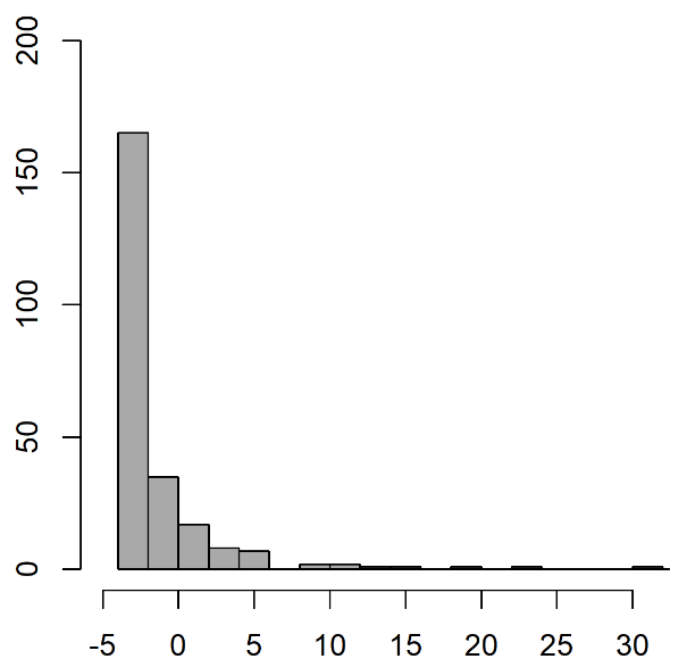

Difference in AIC (uniform model - mixture model)
Non-Recurring Cue

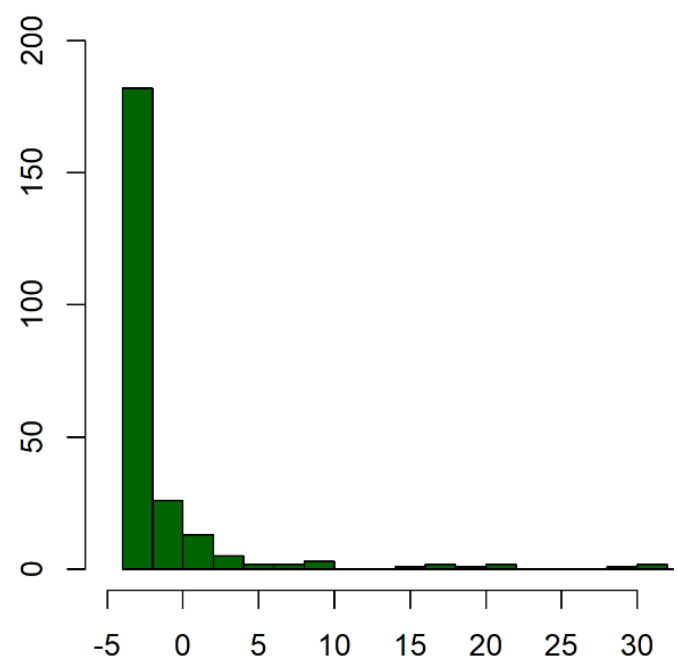

Difference in AIC (uniform model - mixture model)

Note. Positive values show an advantage of the mixture model above the uniform model and vice versa.

\section{Discussion}

Consistent with Experiment 1, the results of Experiment 2 yielded evidence for a very small proportion of guessing-awareness responses. The probability to be in the guessingawareness distribution was estimated to be lower than what would be expected from a slot model, if people always knew right away that they cannot retrieve the correct response in trials where the target was not held in a slot. Consistent with the retrieval-outcome hypothesis, the results therefore suggest that people always try to retrieve what they have in their VWM and make a metacognitive judgement about it based on the outcome of that retrieval effort. 
Additionally, the results suggest that familiarity of the retrieval cue does not affect how people make metacognitive judgements in VWM. Although the retrieval cue was non-recurring and therefore less familiar in half of the trials, participants behaved in the same way as in the recurring-cue condition: On most trials, they engaged in a response search before giving a response, even when they were low in confidence.

One could object that our familiarity manipulation was not strong enough because both the recurring and the non-recurring cues were encoded right before the test. Consequently, even the non-recurring cues were most likely recognized by the participants. This contrasts with the original study that provided evidence for the two-stage theory for JOLs (Son \& Metcalfe, 2005). In the original study, participants engaged in a LTM test and therefore were presented with the retrieval cues after a significantly longer delay. It is possible that in that study participants sometimes even failed to remember the retrieval cue at all. In contrast, in our experiments all cues were presented just before the test and therefore were most likely recognized by the participants. ${ }^{4}$ It could therefore be that our retrieval cues (even the non-recurring cues in Experiment 2) always exceeded the familiarity threshold and that we therefore did not find evidence for the guessing-awareness distribution. Even though this is a crucial difference between the studies, it does not invalidate our conclusion. Naturally, in a WM test the retrieval cues will be presented with just a short (or even no) temporal break before the test. Thus, they will likely be on average more familiar than retrieval cues in a LTM test. This increased familiarity of the retrieval cue, however, is an inherent property of the WM system and not an artefact of the experimental operationalization. It is well possible that people never enter the

${ }^{4}$ This assumption could not be made in the context of a slot model in which there is no memory at all for objects not accommodated in a slot - not even familiarity. 
quick guessing state in WM tests because of this high familiarity of the retrieval cues. However, this just means that people need to engage in a retrieval attempt to know that an information cannot be retrieved from WM.

Finally, there is one further concern that can be raised regarding Experiment 1 and 2: Both experiments build on the assumption that people will not spend time on the task when they know right away that they will not be able to retrieve the response. Even though this assumption seems plausible, we have not empirically demonstrated that people select the first random color when being immediately aware of their inability to retrieve a target feature. It is possible, even if not very likely, that participants always aim to do the color reproduction task in the most conscientious manner, and therefore spend some minimum amount of time on each trial despite already knowing from the start about their inability to retrieve the information. To test this possibility, we conducted Experiment 3.

\section{Experiment 3}

The aim of the following experiment was to provide a test for our assumption that people do not engage in a response search if they know right away that they will not be able to retrieve a target feature. To test this in Experiment 3, we implemented a new-cue condition in which participants were cued with objects at test that were not previously presented during the encoding phase. We assumed that (especially when presented with just two objects) participants will directly realize that the cued object is new and that a retrieval attempt will be futile. Based on our assumption participants will then select the first random color and not engage in a search behavior in the wheel. 


\section{Method}

Participants. 30 students from the University of Zurich participated in this online experiment for either 7.50 CHF or partial course credit. Only students between the age of 18-35 years and without any impairment of their color vision were recruited.

Materials and Procedure. The experiment was programmed in lab.js (Henninger et al., 2019). Participants completed 3 practice trials, followed by 150 experimental trials. The experimental procedure resembled the procedure of Experiment 2: Again, participants were presented with either two, four or eight colored objects for the duration of $3000 \mathrm{~ms}$. In 105 of the trials, participants were cued with one of the presented objects at test (presented cue condition). In 45 of the trials, participants were cued with an object at test that was not shown during the encoding phase (new cue condition). Before the start of the experiment, participants were informed that they will sometimes be cued with new objects during test and instructed to then select whichever color they want and proceed with the experiment. When giving their confidence ratings, participants could either use the slider (which was the same as in the previous experiments) or indicate that the object is new by clicking with the mouse on the corresponding box. 


\section{Results}

Descriptives. Table 3 shows the average frequency in which participants selected the response "object is new". There were 15 new objects per set size condition. On average, most new objects were identified as such by the participants. However, with increasing set size participants made more mistakes. In contrast, objects in the presented cue conditions were only rarely labeled as new objects (only 7 out of 105 objects on average), and this happened mostly in the more difficult set size 8 condition. Taken together, participants were good at detecting the new, not previously presented objects and labeled them as such.

\section{Table 3}

Average Frequency of the "object is new" response as a Function of Set Size and Cue Condition

\begin{tabular}{ccc}
\multicolumn{2}{l}{ Function of Set Size and Cue Condition } \\
\hline cue & set size & average frequency \\
\hline \multirow{3}{*}{ presented } & 2 & 0.37 \\
& 4 & 1.5 \\
\multirow{4}{*}{ new } & 8 & 5.1 \\
& 2 & 13.13 \\
& 4 & 11.8 \\
& 8 & 9.7 \\
\hline
\end{tabular}

Figure 12 shows the average search angle in both cue conditions as a function of confidence. In contrast to the two previous experiments, we can see here that the average search angle was far below a random angle of 90 when participants indicated that a cue was a "new object". It did not play a role whether the object was indeed new or whether it was an encoded object: When the participants thought that they have not seen the object before, they just quickly selected a random color.

As in the previous experiment, the search angle data of the presented cue condition was (close to) 90 for the objects that were not identified as "new" - even when the confidence ratings 
were rather low. As expected, and already implied by Table 3 there were not many observations in which participants gave high confidence responses to new objects, explaining the large confidence intervals in this condition.

\section{Figure 12}

Search Angle as a Function of Confidence and Cue.

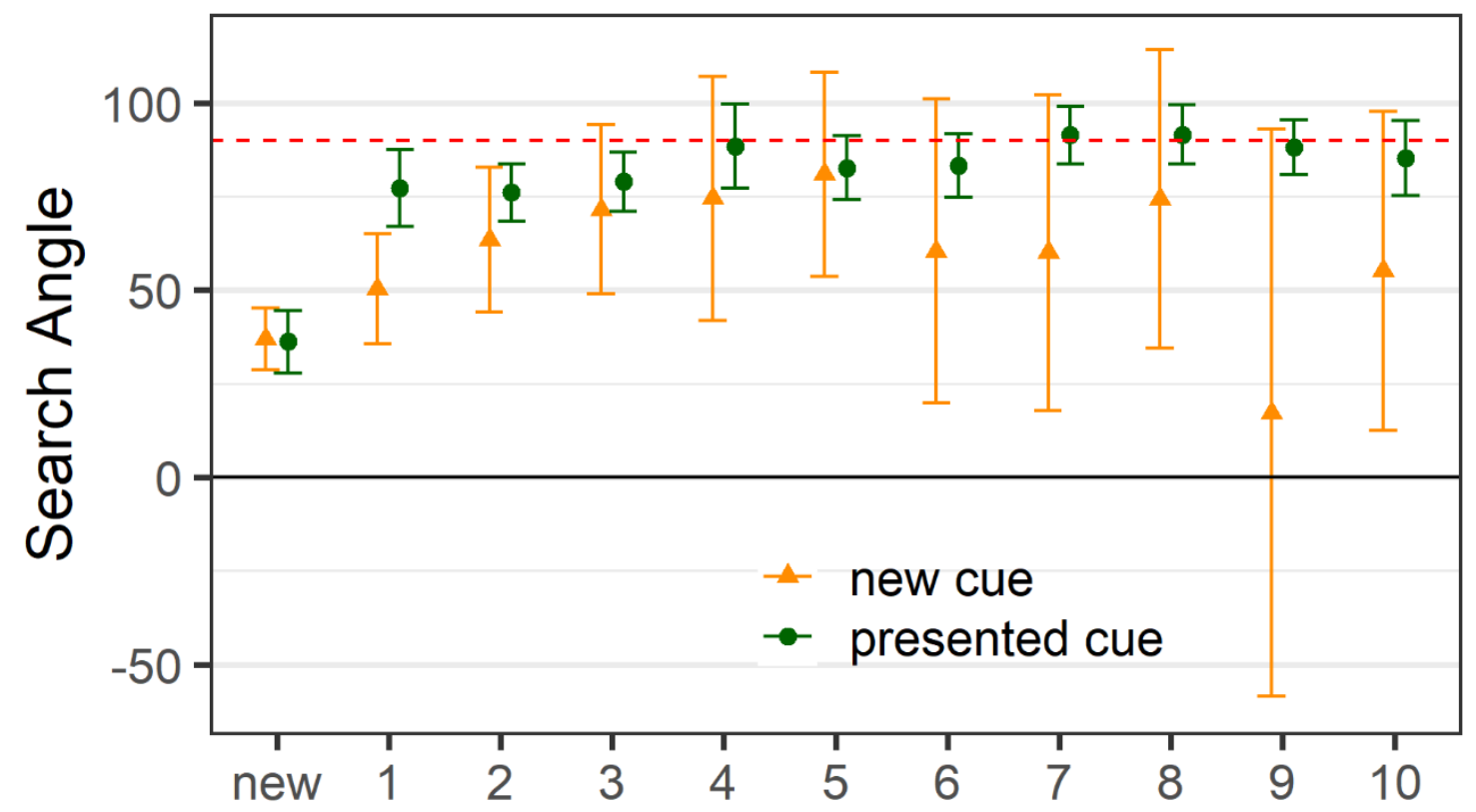

\section{Confidence}

Note. Error bars represent 95\% within-subjects confidence intervals. The red dashed line marks the average search angle under the assumption that responses are at random distance from the first color. The black line marks a search angle of 0 , the natural lower boundary of the search angle scale. The continuous confidence scale was binned in 10 bins ranging from 1 (very low in confidence) to 10 (very high in confidence).

Modelling. To model the data, we proceeded similarly as in Experiment 1 and 2. Table 4 shows the results of the hierarchical modelling approach. In the presented cue condition, the WAIC difference was negative for set size two, but positive for set size four and eight. This 
reflects an advantage of the uniform model over the mixture model in the set size two condition.

Thus, the data of the set size two condition is better described through just the uniform retrievalattempt distribution. In contrast, the data of the higher set sizes is better described through a mixture of the retrieval-attempt and the guessing-awareness distribution. However, the estimates for $P_{g}(1.4 \%-18.1 \%)$ were again much lower than what would be expected from a slot model, if participants guessed right away on all trials in which they did not have the target item in a slot. The $P_{g}$ estimates for the new cue condition, however, differed substantially: Here the probability to be in the guessing awareness distribution ranged from $58.4 \%-75.5 \%$, reflecting participants' tendency to quickly guess and not spend time in the grey wheel. The positive and bigger WAIC difference also supports the advantage of the mixture model over the uniform model in all set sizes of the new cue condition.

\section{Table 4}

Mean Posterior Parameter Estimates of the Bayesian Hierarchical Adapted Mixture Model, WAIC for the Adapted Mixture Model (WAIC $\left.C_{m i x}\right)$, the Uniform Model (WAIC $\left.C_{\text {uni }}\right)$

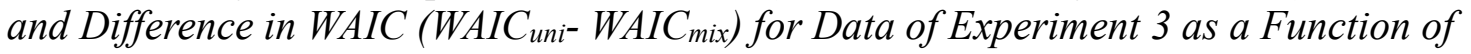
Set Size and Cue Condition

\begin{tabular}{|c|c|c|c|c|c|c|}
\hline cue & $\begin{array}{l}\text { set } \\
\text { size }\end{array}$ & $P_{g}$ & $K$ & $\mathrm{WAIC}_{\text {mix }}$ & WAIC $_{\text {uni }}$ & $\begin{array}{l}\text { WAIC }_{\text {uni- }} \\
\text { WAIC }_{\text {mix }}\end{array}$ \\
\hline \multirow[t]{3}{*}{ presented } & 2 & $\begin{array}{c}.014 \\
{[0, .036]}\end{array}$ & $\begin{array}{c}9.14 \\
{[3.0,23.34]}\end{array}$ & 3862.3 & 3859.54 & -2.76 \\
\hline & 4 & $\begin{array}{c}.108 \\
{[.048, .171]}\end{array}$ & $\begin{array}{c}5.82 \\
{[3.0,10.65]}\end{array}$ & 3820.14 & 3859.54 & 39.4 \\
\hline & 8 & $\begin{array}{c}.181 \\
{[.115, .25]}\end{array}$ & $\begin{array}{c}8.38 \\
{[3.0,15.82]}\end{array}$ & 3706.08 & 3859.54 & 153.46 \\
\hline \multirow[t]{3}{*}{ new } & 2 & $\begin{array}{c}.755 \\
{[.616, .902]}\end{array}$ & $\begin{array}{c}7.61 \\
{[3.0,12.8]}\end{array}$ & 879.48 & 1654.09 & 774.61 \\
\hline & 4 & $\begin{array}{c}.584 \\
{[.481, .69]}\end{array}$ & $\begin{array}{c}9.4 \\
{[3.0,14.83]}\end{array}$ & 1130.6 & 1654.09 & 523.49 \\
\hline & 8 & $\begin{array}{c}.635 \\
{[.538, .726]}\end{array}$ & $\begin{array}{c}5.48 \\
{[3.0,8.89]}\end{array}$ & 1177.03 & 1654.09 & 477.06 \\
\hline
\end{tabular}


Note. Values in brackets represent the boundaries of the $95 \%$ highest density interval for the parameters $P_{g}$ and $K$.

As in the previous experiments, we also fitted both models to the data of each participant separately. As expected, the $P_{g}$ parameter differed here as well between the two cue conditions. Whereas the probability to be in the guessing awareness distribution was again rather low in the presented-cue condition, it reached a value above 0.75 in the new-cue condition when two objects were presented. As we assumed, participants thus engaged in quick guesses in the majority of the set size two trials when being cued with an object that was not previously encoded. This probability was lower for higher set sizes - probably because it is more difficult to spot a new object there. However, even in the set size eight condition participants abbreviated their search in more than $60 \%$ of the trials. 


\section{Figure 13}

Estimates of the Probability to be in the Guessing-Awareness Distribution $\left(P_{g}\right)$ as a Function of Set Size and Cue Condition in Experiment 3.

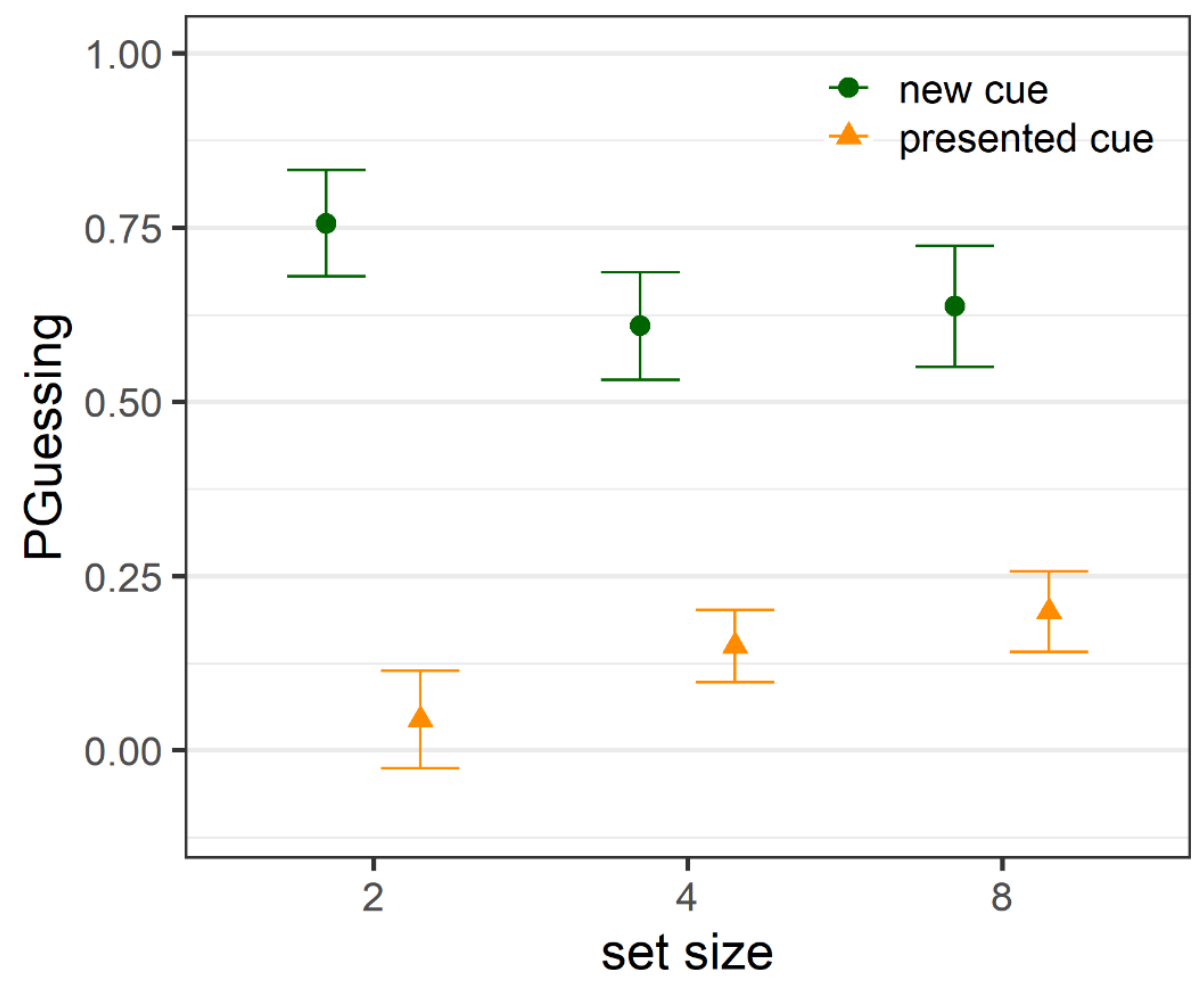

Note. Error bars represent $95 \%$ within-subjects confidence intervals.

The same pattern becomes evident when comparing the AIC between the mixture model and the uniform model for each participant in the presented cue and new cue condition. Consistent with Experiment 1 and 2, there is only sparse evidence for the existence of the guessing-awareness distribution in the presented cue condition (see Figure 14). Again, the AIC for the uniform model indicated a better fit than the AIC for the mixture model for the majority of participants.

However, for the new-cue condition the AIC comparison yielded a different result. Here, the AIC difference is positive for most participants, indicating an advantage of the mixture model over 
the uniform model. This analysis therefore provides strong evidence for the existence of the guessing-awareness distribution in the new-cue condition.

\section{Figure 14}

Frequencies of AIC Differences in the Presented Cue Condition (left) and New Cue Condition (right).

\section{Presented Cue}

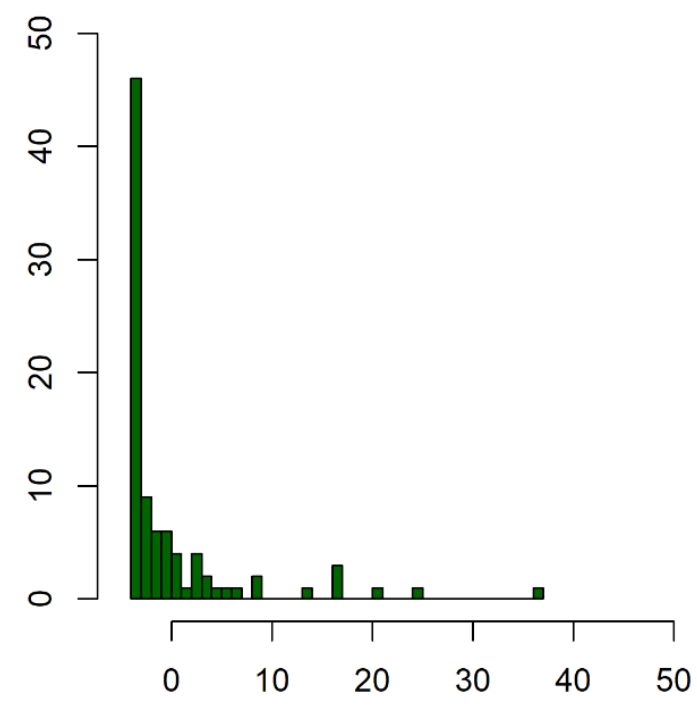

Difference in AIC (uniform model - mixture model)
New Cue

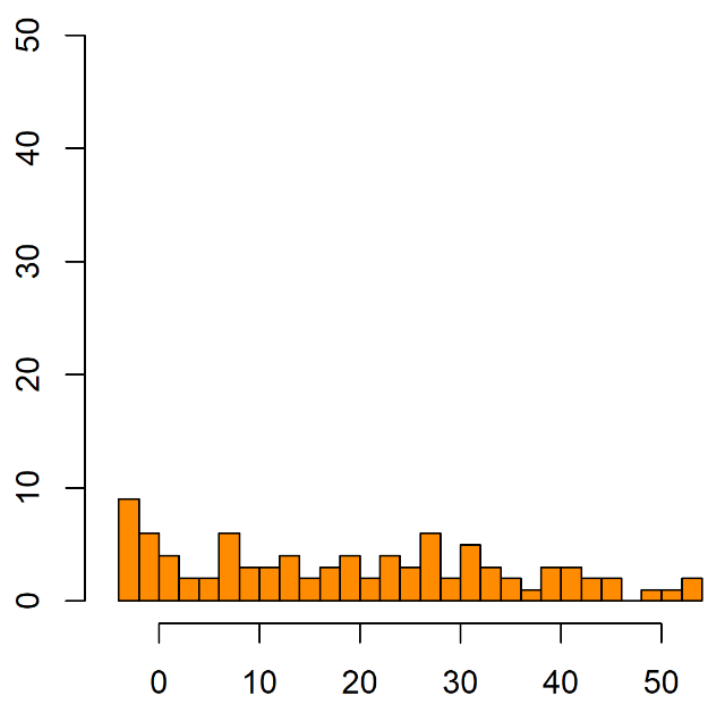

Difference in AIC (uniform model - mixture model)

Note. Positive values show an advantage of the mixture model above the uniform model and vice versa.

\section{Discussion}

The results of Experiment 3 show that people tend to select the first random color when they realize that the object is new and therefore, it is not possible to retrieve the color from memory. These results provide support for our assumption that people do not search for a response in the grey wheel when they know that this behavior will be futile. This finding is important with regards to the interpretation of Experiment 1 and 2 where we found only little evidence for the selection of the first random response. This is because it suggests that - in 
contrast to Experiment 3 - participants of Experiment 1 and 2 were not aware of their inability to retrieve the target feature right away.

\section{Experiment 4}

Based on response search data, the first three experiments provided first evidence in favor of the retrieval-outcome hypothesis for confidence ratings in VWM. To be able to make stronger conclusions about how and when people decide that they do not remember an information, we aimed to test the same hypothesis using a different approach in Experiment 4. Many prior studies with the focus on guessing in LTM recognition tasks used RTs as a measure to argue for or against the retrieval-outcome hypothesis (Benjamin et al., 1998; Son \& Metcalfe, 2005). The rationale behind that is that longer RTs for low-confidence responses (as compared to high confidence responses) are more consistent with the retrieval-outcome hypothesis, whereas significantly shorter RTs for low-confidence responses provide some evidence for the existence of a prior, quickly operating monitoring stage detecting the absence of information in memory. Whereas it is reasonable to use RTs in recognition tasks to draw conclusions about the underlying cognitive (and metacognitive) processes, the interpretation of RTs in the color reproduction task is less straightforward. This is because longer RTs in the color reproduction task cannot solely be attributed to a longer retrieval process, but also to an attempt to adjust the selected color as precisely as possible to the memory representation. Relatively long RTs could therefore reflect on the one hand, engaging in a long unsuccessful retrieval attempt due to a lack of memory, but on the other hand also having a very precise representation of the color in mind and spending much time on finely adjusting one's response. A pure comparison of RTs for low confidence and high confidence responses in the color reproduction task would therefore not help distinguishing between different ideas about metacognitive processes. 
Instead of comparing the RTs of low and high confidence responses directly, we analyzed RTs for the color reproduction task and for the confidence judgements depending on the order of these two assessments in Experiment 4. The idea was the following: If retrieval is a necessary part of the generation of confidence judgements - as proposed by the retrieval-outcome hypothesis - then RTs for confidence judgements should be shorter if they follow the color reproduction task as compared to when they precede it. This is because participants have already made the retrieval experience and only have to translate it into a confidence judgement. When confidence judgements are assessed prior to the color reproduction task, in contrast, participants must first engage in a retrieval attempt and then additionally translate the information gained through the retrieval process to a corresponding confidence judgement, leading to longer RTs. The same argument applies to the RTs for the color reproduction task: If retrieval is a necessary part of the generation of confidence judgements, RTs for the color reproduction task should be shorter if it is performed after the confidence judgements, because participants then only have to select the already retrieved response on the wheel. In contrast, if making a judgement about one's confidence in a response does not require retrieval of the color, RTs for both measures should not be affected by the order of the color reproduction task and the assessment of confidence. 


\section{Method}

Participants. Twenty students from the University of Zurich participated in this lab experiment for either $30 \mathrm{CHF}$ or partial course credit. Again, only students aged 18-35 years without any impairment of the color vision were recruited for the experiment.

Materials and Procedure. The experiment was programmed in MATLAB using the Psychophysics Toolbox extension. Each participant took part in two 1-hour sessions and completed overall 648 experimental trials, 324 trials per session. The time between participation in session 1 and session 2 ranged from one day to up to a week. Overall, the procedure resembled the procedure of the previous experiments with two major modifications: Instead of the grey wheel, a traditional color wheel was used, where the colors are visible right away. As in Experiment 1 and 2, the wheel randomly rotated on each trial. Additionally, the order of the color reproduction task and the assessment of confidence was varied within-subjects between the two sessions. Half of the participants performed the "color reproduction task first + confidence ratings second" in the first session and "confidence rating first + color reproduction task second" in the second session. For the other half of participants, the session order was reversed. Both sessions started with 3 practice trials. 


\section{Results}

RT data for the color reproduction task as well as for the assessment of confidence was analyzed with a BANOVA using the anovaBF function from the R-package BayesFactor (Morey \& Rouder, 2018). In the specified BANOVAs, individual mean RT in each condition was predicted by the factors set size and order condition. The factor participant was included as a random effect. The Bayes factors denoted $\mathrm{BF}_{10}$ quantify the amount of evidence for the effects of interest. The Bayes factor denoted $\mathrm{BF}_{01}=1 / \mathrm{BF}_{10}$ quantify the evidence against the tested effect.

\section{Figure 15}

Mean Reaction Times for the Color Reproduction Task and the Assessment of Confidence Ratings in Experiment 3 as a Function of Set Size and Order of Tasks.
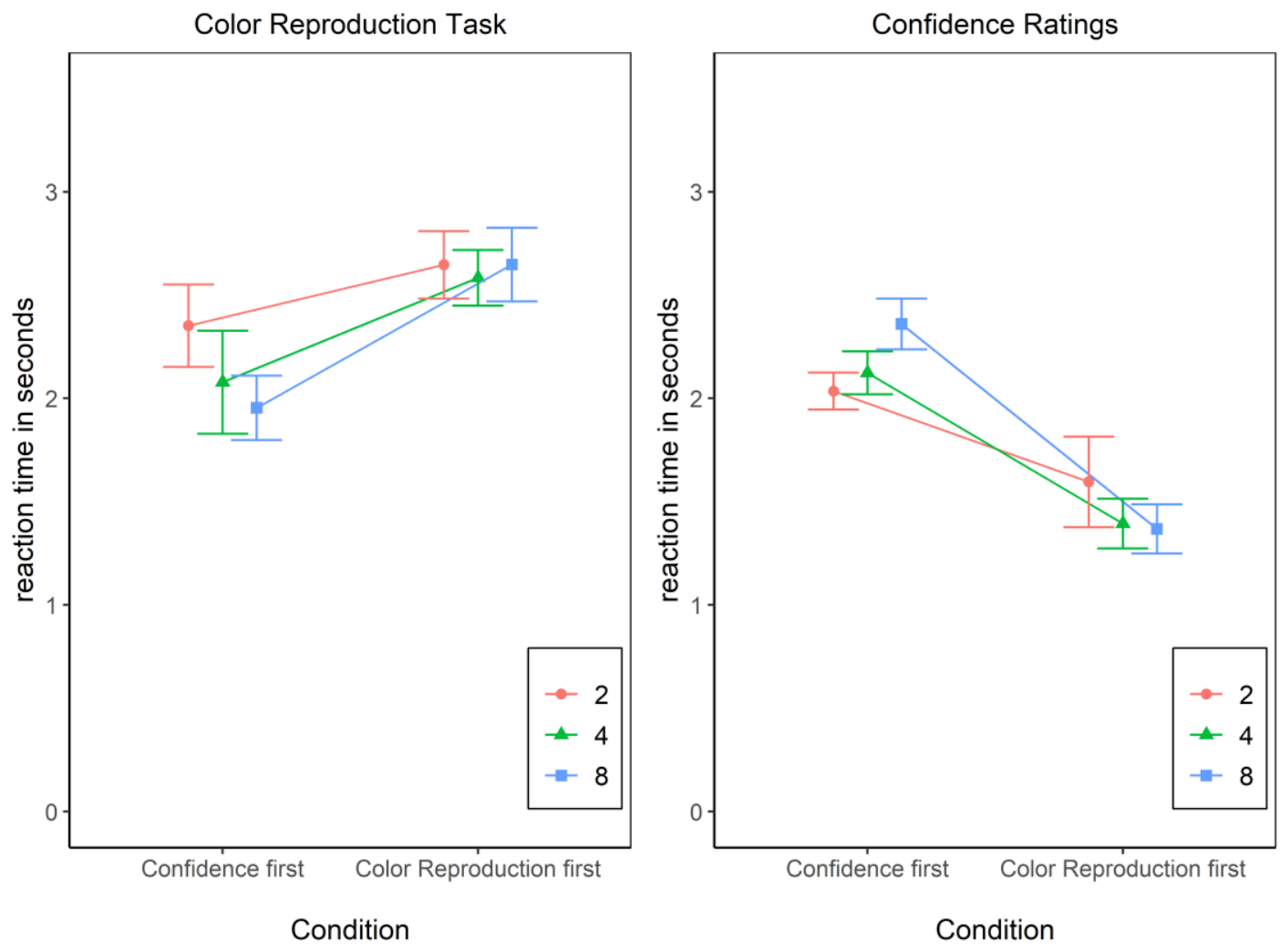

Note. Error bars represent 95\% within-subjects confidence intervals. 
Color Reproduction Task. As depicted in Figure 15, there was a credible main effect of order of tasks on RTs in the color reproduction task, $\mathrm{BF}_{10}=2.94 \times 10^{31}$, which is consistent with the retrieval-outcome hypothesis. There was also a credible main effect of set size on RTs, $\mathrm{BF}_{10}=11.25:$ On average, participants showed longer RTs in set size two as compared to set size four and eight - which could be due to the fact that the memory of the color was more precise in set size two and therefore, participants spent more time searching for the precise shade of the color in the wheel. The effect of set size was not similarly large in both order conditions, which was reflected in an interaction between set size and order of tasks on $\mathrm{RTs}_{\mathrm{s}} \mathrm{BF}_{10}=6.79$.

Confidence Ratings. For the RTs for the confidence ratings there was evidence against the presence of a main effect of set size, $\mathrm{BF}_{01}=74.2$. Consistent with the results of the analysis of RTs in the reproduction task, there was evidence for a main effect of order of tasks on RTs for the confidence ratings, $\mathrm{BF}_{10}=9.46 \times 10^{78}$. Giving a confidence rating took longer when confidence was assessed first than when they followed the color-reproduction task (2.17 vs. 1.45 $\mathrm{s})$. The interaction between order of task and set size, $\mathrm{BF}_{10}=1.85 \times 10^{5}$, reflects the fact that the difference in RTs between the order conditions was larger with increasing set size.

\section{Discussion}

The results of Experiment 4 show that the order of the assessment of confidence and color reproduction influences RTs for both tasks. This finding suggests that these two tasks are not independent of each other but share one component that can be re-used by whichever response was given second. Based on prior findings from the literature and the findings of Experiment 1 to 3, it is reasonable to assume that this common component is the attempt to retrieve the color from memory. 
One could object that the first task only took more time because participants required some additional time to get started with the "active" part of the trial. One reason for that could be that participants sometimes take their hand away from the mouse while encoding the colors and therefore needed some time to put it back to the mouse for providing their responses. Unfortunately, we did not observe the participants during the task and therefore cannot entirely rule out this alternative explanation. However, one would assume that such a behavior would lead to very much longer RTs as compared to the trials were participants kept the mouse in their hand. To test whether such extremely longer RTs were responsible for the obtained RT pattern, we repeated the same analyses excluding those extreme values (RTs above 3 standard deviations above the mean) and still found the same pattern. Additionally, taking the hand systematically away from the mouse for a couple of seconds at encoding, just to put it back again to give a response, would be a very inefficient behavior, especially when performing more than 300 trials. We therefore do not consider this alternative explanation to be very likely.

One could further object that even if participants kept the mouse in their hand, they required some time to get started with giving the responses after the encoding phase. This argument resembles what is commonly discussed as task-switching costs (Monsell, 2003). As the term implies, task-switching costs are costs that occur when people switch between two different tasks. These costs, however, would not only occur when starting with the first task, but also when switching between the first and the second task. Therefore, task-switching cost itself cannot explain longer RTs for the first task. Another related finding is that starting to retrieve items from memory comes with a retrieval-initiation costs. This is reflected in longer RTs for the first reported item as compared to the subsequently reported items of a memory set (Anderson \& Matessa, 1997; Maybery et al., 2002). If we assume that only the color reproduction task requires 
retrieval from memory, retrieval-initiation costs should affect the color reproduction task independent of order. This is in contrast to the shorter RTs that we observed in the color reproduction task when it was preceded by confidence reports. However, if we assume that confidence judgements involve retrieval, our findings are consistent with the literature on retrieval-initiation cost: Because retrieval has been initiated (and completed) already at the assessment of confidence, no retrieval-initiation costs occur anymore at the color reproduction task.

\section{General Discussion}

In four experiments using two novel approaches we tested whether people have an instant knowledge about their inability to retrieve a good approximation to a target feature from VWM, or whether they need to engage in an unsuccessful retrieval attempt to conclude that they do not remember it well. In Experiments 1, we investigated whether people made use of the opportunity to enter a quick guess rather than engaging in a lengthy search of a particular response in the grey wheel. Model comparisons provided evidence for the existence of quick guesses, but only in a very small percentage of trials - speaking against a general, instant awareness of the inability to retrieve a color. A similar pattern was observed in Experiment 2, where we varied the familiarity of the retrieval cue but did not find any influence of this manipulation on participants' search behavior. Again, participants engaged in a response search on most trials, even when they were low in confidence, and independent of the familiarity of the retrieval cue. In Experiment 3 we tested our assumption that people will not engage in a response search in the grey wheel when they know that this behavior is futile. Therefore, we cued participants with objects during the test that were not presented during encoding. In this case people tended to select the first color that appears in the grey wheel, confirming our assumption. Finally, in Experiment 4, we varied the 
order of the confidence ratings and the reproduction of the color. The idea was that order should not matter for RTs if the two tasks do not have any process in common. However, consistent with the retrieval-outcome hypothesis, RTs were much shorter for whichever task came second.

One major difference between our study and the original study of Son and Metcalfe (2005) is that we focused on WM, and hence, our retrieval cues were presented right before the color reproduction test. It is reasonable to assume that the retrieval cues in our study were therefore on average more familiar to the participants than the retrieval cues in the original study. This higher familiarity of the retrieval cues - which is an inherent property of a WM test - can prevent people from ever entering the first, quick guessing state that the authors described. If so, it is possible that the mechanisms behind metacognitive monitoring are similar in WM and LTM, but that the increased familiarity of retrieval cues causes people to always engage in a retrieval attempt when they monitor their WM. Even if our data does not provide any information on whether or not people first evaluate the familiarity of the retrieval cue, it clearly shows that quick guesses are a rare exception. It is much more common that people engage in a retrieval attempt to judge what they can (and cannot) retrieve from WM.

Whereas the retrieval-outcome hypothesis can explain most of the observed data, it fails to explain why in Experiment 1 and 2 there was evidence for a small number of quick guesses at all. The most straightforward explanation for these quick guesses is a prior, retrieval-independent monitoring stage that was already proposed in the literature on LTM monitoring. However, quick guesses occurred very rarely, and independent of the familiarity of the retrieval cue, which is in contrast to the two-stage process theory proposed by Son and Metcalfe (2005). Moreover, our data does not offer any systematic patterns that could explain why some people sometimes enter this stage and others do not. Future research is therefore needed to provide more evidence in 
favor of or against such a prior monitoring stage. A more parsimonious explanation for these quick responses that does not assume a prior stage of monitoring is that on some trials people accidently clicked on a color prematurely, when the mouse was still close to its initial entry point into the wheel. Accidental clicks can also explain why the average search angle was below 90 not only in low-confidence trials, but also in trials were people indicated to be higher in confidence. However, one would expect that those accidental clicks occur similarly often in all set size conditions, which is not the case in our data (especially not in the data of Experiment 2). Accidental clicks therefore might have increased the number of quick guesses but fail to account for them fully. Another explanation for those quick guesses is that some participants consciously take a shortcut in their metacognitive monitoring process when they believe that the target feature is most likely poorly remembered. More specifically, participants know that they are bad at remembering eight colors, and therefore immediately give up retrieving and reproducing the studied color accurately. This explanation is in line with the retrieval-outcome hypothesis because it implies that participants do not know how poorly they remember something without a prior retrieval attempt. However, they can infer from the study situation (seeing eight colors) and prior experience (having failed to retrieve eight colors) that the information must be weakly remembered and therefore intentionally skip the retrieval attempt and give a quick guess to save some time.

It is possible that participants moved the cursor around the wheel because they hoped to recognize the correct color when they see it. Such a behavior would be reasonable as long as people believe that they have at least a faint memory trace of the target. It would not make sense, however, if participants know right away that the target feature is not in their memory at all. And indeed, we did not observe a search behavior in Experiment 3 where we cued with completely 
new objects. Therefore, our results are compatible with the assumption that participants often find themselves in a meta-cognitive state of having very little information about the target in memory, but they are not compatible with the assumption that they frequently experience a state with no information at all about the target.

The distinction between a memory state with very poor information and a memory state with no information at all about the target lies at the heart of a theoretical debate about the nature of the WM capacity limit. Whereas slot models assume that, at high set sizes, people often have no information about the target (because the target is not held in a slot), resource models and interference models assume that they never have strictly zero information about the target. Our findings concerning corresponding meta-cognitive states therefore can be related to the question about actual memory states. The finding that people consistently search for a color in the grey wheel suggests that people are not metacognitively aware of being in a zero-information state about the target. This, in turn, could either mean that, contrary to slot models, no zeroinformation states exist in VWM, or else, that people are not aware when they are in a zeroinformation state. As already mentioned in the introduction, our data cannot decisively rule out any of the currently discussed models of WM. However, the finding that people engage in a lengthy search process on most trials (even if they end up committing a large reproduction error) is more in line with resource or interference theories of VWM, and less compatible with slot theories of VWM that assume the existence of a zero-information state. At the very least, the data clearly suggests that if a zero-information state exists, people are commonly not aware of being in it without trying - and failing - to retrieve the target feature first. 
Taken together, the results of all four experiments suggest that a retrieval attempt is a crucial part of metacognitive monitoring in VWM - even when the target feature is poorly remembered.

\section{References}

Anderson, J. R., \& Matessa, M. (1997). Production System Theory and Serial Memory. Psychological Review, 104(4), 728-748. http://act-

r.psy.cmu.edu/publications/pubinfo.php?id=29

Begg, I., Duft, S., Lalonde, P., Melnick, R., \& Sanvito, J. (1989). Memory predictions are based on ease of processing. Journal of Memory and Language, 28(5), 610-632. https://doi.org/10.1016/0749-596X(89)90016-8

Benjamin, A. S., Bjork, R. A., \& Schwartz, B. L. (1998). The Mismeasure of Memory: When Retrieval Fluency Is Misleading as a Metamnemonic Index. Journal of Experimental Psychology: General, 127(1), 55-68. https://doi.org/10.1037/0096-3445.127.1.55

Brady, T. F., Konkle, T., Gill, J., Oliva, A., \& Alvarez, G. A. (2013). Visual Long-Term Memory Has the Same Limit on Fidelity as Visual Working Memory. Psychological Science, 24(6), 981-990. https://doi.org/10.1177/0956797612465439

Brainard, D. H. (1997). The Psychophysics Toolbox. Spatial Vision, 10(4), 433-436. https://doi.org/10.1163/156856897X00357

Cowan, N. (2001). The magical number 4 in short-term memory: A reconsideration of mental storage capacity. Behavioral and Brain Sciences, 24(1), 87-114. https://doi.org/10.1017/S0140525X01003922

Donkin, C., Nosofsky, R. M., Gold, J. M., \& Shiffrin, R. M. (2013). Discrete-Slots Models of 
Visual Working-Memory Response Times. Psychological Review, 120(4), 873-902. https://doi.org/10.1037/a0034247.Discrete-Slots

Double, K. S., Birney, D. P., \& Walker, S. A. (2018). A meta-analysis and systematic review of reactivity to judgements of learning. Memory, 26(6), 741-750. https://doi.org/10.1080/09658211.2017.1404111

Fougnie, D., Suchow, J. W., \& Alvarez, G. A. (2012). Variability in the quality of visual working memory. Nature Communications, 3. https://doi.org/10.1038/ncomms2237

Henninger, F., Shevchenko, Y., Mertens, U. K., Kieslich, P., \& Hilbig, B. (2019). lab.js: A free, open, online study builder. 6. https://doi.org/10.31234/osf.io/fqr49

Kelley, C. M., \& Lindsay, D. S. (1993). Remembering Mistaken for Knowing: Ease of Retrieval as a Basis for Confidence in Answers to General Knowledge Questions. In Journal of Memory and Language (Vol. 32, Issue 1, pp. 1-24). https://doi.org/10.1006/jmla.1993.1001

Maybery, M. T., Parmentier, F. B. R., \& Jones, D. M. (2002). Grouping of list items reflected in the timing of recall: Implications for models of serial verbal memory. Journal of Memory and Language, 47(3), 360-385. https://doi.org/10.1016/S0749-596X(02)00014-1

Monsell, S. (2003). Task switching. Trends in Cognitive Sciences, 7(3), 134-140. https://doi.org/10.1016/S1364-6613(03)00028-7

Nelson, T. O., \& Dunlosky, J. (1991). When People’s Judgments of Learning (JOLs) are Extremely Accurate at Predicting Subsequent Recall: The "delayed-JOL effect." Psychological Science, 2(4), 267-270. https://doi.org/10.1111/j.1467-9280.1991.tb00147.x

Oberauer, K., \& Lin, H. Y. (2017). An interference model of visual working memory. Psychological Review, 124(1), 21-59. https://doi.org/10.1037/rev0000044

Province, J. M., \& Rouder, J. N. (2012). Evidence for discrete-state processing in recognition 
memory. Proceedings of the National Academy of Sciences of the United States of America, 109(36), 14357-14362. https://doi.org/10.1073/pnas.1103880109

Son, L. K., \& Metcalfe, J. (2005). Judgments of learning: Evidence for a two-stage process. Memory and Cognition, 33(6), 1116-1129. https://doi.org/10.3758/BF03193217

Souza, A. S., Rerko, L., \& Oberauer, K. (2016). Getting more from visual working memory: Retro-cues enhance retrieval and protect from visual interference. Journal of Experimental Psychology: Human Perception and Performance, 42(6), 890-910.

https://doi.org/10.1037/xhp0000192

Van Den Berg, R., Shin, H., Chou, W. C., George, R., \& Ma, W. J. (2012). Variability in encoding precision accounts for visual short-term memory limitations. Proceedings of the National Academy of Sciences of the United States of America, 109(22), 8780-8785. https://doi.org/10.1073/pnas.1117465109

Zhang, W., \& Luck, S. J. (2008). Discrete fixed-resolution representations in visual working memory. Nature, 453(7192), 233-235. https://doi.org/10.1038/nature06860 